\title{
Congruence relations for Shimura varieties associated to some unitary groups
}

\author{
Oliver Bültel \\ Torsten Wedhorn
}

\section{Introduction}

Let $G / \mathbb{Q}$ be a reductive group and $X$ be a $G(\mathbb{R})$-conjugacy class of homomorphisms $\operatorname{Res}_{\mathbb{C} / \mathbb{R}}\left(\mathbb{G}_{m}\right) \longrightarrow G_{\mathbb{R}}$ such that $(G, X)$ is a Shimura datum and denote for some compact open subgroup $K \subset G\left(\mathbf{A}_{f}\right)$ by $\operatorname{Sh}_{K}(G, X)$ the associated Shimura variety. Its $\mathbb{C}$-valued points are given by $G(\mathbb{Q}) \backslash\left(\left(X \times G\left(\mathbf{A}_{f}\right) / K\right)\right.$ and it is defined over a number field $E$ which is associated to $(G, X)$. Hence its cohomology carries an action of $\Gamma_{E}$ (the absolute Galois group of $E$ ) and of the Hecke algebra $\mathcal{H}\left(G\left(\mathbf{A}_{f}\right) / / K\right)$ of $K$-biinvariant locally constant $\mathbb{Q}$-valued functions with compact support, and these actions commute with each other. It is one of the fundamental problems to understand the precise relation of these two actions. One step towards this understanding is the following: Let $p$ be a prime of good reduction (i.e. $G_{\mathbb{Q}_{p}}$ is unramified and we have $K=K_{p} K^{p}$ with $K_{p} \subset G\left(\mathbb{Q}_{p}\right)$ hyperspecial) and let $v$ be a place of $E$ lying over $p$. Denote by $\mathrm{Fr}_{v}$ the conjugacy class of corresponding geometric Frobenius elements in $\Gamma_{E}$. Blasius and Rogawski [BR] have defined a polynomial $H=H_{(G, X), p}$ (the socalled Hecke polynomial) with coefficients in the local Hecke algebra $\mathcal{H}\left(G\left(\mathbb{Q}_{p}\right) / / K_{p}\right)$ and they conjectured that $H\left(\operatorname{Fr}_{v}\right)=0$.

This is known for Shimura curves for a long time by the work of Eichler/Shimura and Ihara. Further, Faltings and Chai $[\mathrm{FC}]$ have proved in the Siegel case that $\operatorname{Fr}_{v}$ satisfies an equation of the correct degree. Further the first of us has proved the conjecture in the case of certain orthogonal groups [??], and the second in the PELcase when $G_{\mathbb{Q}_{p}}$ is a split reductive group [We2].

The goal of this article is to prove this conjecture in the case where $G$ is the group of $B$-linear similitudes of a skew-hermitian space $(V,\langle\rangle$,$) where B$ is a simple $\mathbb{Q}$-algebra endowed with a positive involution which is split at the chosen prime $p$ which we assume to be odd. We further suppose that $G_{\mathbb{R}}$ is isomorphic to a group auf unitary similitudes of a hermitian form of signature $(n-1,1)$. We finally assume 
that $n$ is even (in particular, the case of Picard modular surfaces is excluded). In fact, we also assume that $B_{\mathbb{Q}_{p}}$ is simple, as otherwise we are in the situation of [We2]. See chapter 1 for the precise assumptions.

As we are in the PEL-case, $\operatorname{Sh}_{K}(G, X)$ can be written as a moduli space of abelian varieties with certain additional structures and it has a model $\mathcal{M}$ over $O_{E_{(p)}}$. To prove the congruence relation we proceed in three steps: First we study the reduction of $\mathcal{M}$ to characteristic $p$. In the second part we examine a moduli space of isogenies which is used to define the Hecke correspondence and an extension in characteristic $p$. In the final chapter we use the obtained results to prove the congruence relation.

We are now going to describe the results of the three parts in more detail. For this we assume only that the signature is of the form $(n-1,1)$, but $n$ can be arbitrary (the assumption " $n$ even" is needed only in the last chapter).

In the first part (chapter $3-5$ ) we look at three stratifications of the special fibre $\mathcal{M}_{0}$ of $\mathcal{M}$. These stratifications are defined as follows: Geometric points of $\mathcal{M}_{0}$ are given by isomorphism classes of tuples $(A, \iota, \lambda, \bar{\eta})$ where $A$ is an abelian variety, $\iota$ is an action of an order $O_{B}$ of $B, \lambda$ is a $p$-principal polarization, and $\bar{\eta}$ is a level structure. The strata are given by

(a) The isomorphism class of the isocrystal associated to $(A, \iota, \lambda)$ (the Newton polygon stratification),

(b) The isomorphism class of the $p$-torsion of $(A, \iota, \lambda)$ (the Ekedahl-Oort stratification),

(c) The isomorphism class of the $p$-divisible group of $(A, \iota, \lambda)$ (the final stratification).

Our main result in this part is the following

Theorem: On the non-supersingular locus of $\mathcal{M}_{0}$ the three stratifications coincide.

This implies that every Newton polygon stratum is a union of Ekedahl-Oort strata and as a corollary we get from the corresponding fact for the Ekedahl Oort stratification [We3] that the closure of a Newton polygon stratum is again a union of Newton polygon strata and that all Newton polygon strata are equi-dimensional, nonempty and of the expected dimension. In particular we see that the supersingular locus $\mathcal{M}^{\text {ss }}$ has dimension strictly smaller than half of the dimension of $\mathcal{M}_{0}$ if and only if $n$ is even (if $n$ is odd, we have $\operatorname{dim}\left(\mathcal{M}^{\text {ss }}\right)=\operatorname{dim}\left(\mathcal{M}_{0}\right) / 2$ ).

In the second part (chapter 6 and 7 and proposition 8.7) we look at the special fibre $p$ - Isog $_{0}$ of the moduli space of $p$-isogenies $f:(A, \iota, \lambda, \bar{\eta}) \rightarrow\left(A^{\prime}, \iota^{\prime}, \lambda^{\prime}, \bar{\eta}^{\prime}\right)$. Here 
our main results are

- The canonical morphisms source and target $p$-Isog $\longrightarrow \mathcal{M}_{0}$ are finite over the non-supersingular locus and they are finite locally free over the generic stratum (with respect to any of the three stratifications).

- We describe $p$-Isog 0 generically using an analogue of the local model which we call "generic model". In particular we find that every component of $p$-Isog has $_{0}$ dimension greater or equal than $\operatorname{dim}\left(\mathcal{M}_{0}\right)$.

- Let $p$-Isog ${ }^{\mu}$ be the inverse image of the generic stratum of $\mathcal{M}_{0}$ in $p$-Isog . Then every geometric point of $p$-Isog ${ }^{\mu}$ admits a canonical lift to characteristic zero.

As a corollary we get that $p$-Isog ${ }^{\mu}$ is dense in $p$-Isog 0 if and only if $n$ is even

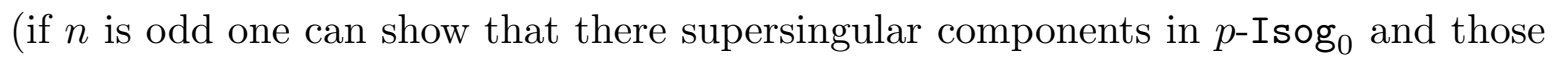
components are even in the closure of the generic fibre of $p$-Isog).

In the third part (chapter 8) we prove the principal result. Here we assume that $n$ is even. The main idea of the proof is the same as in [FC] and [We2]. We consider the $\mathbb{Q}$-vector space $\mathbb{Q}[p$-Isog $\otimes E]$ (resp. $\mathbb{Q}\left[p\right.$-Isog $\left.{ }_{0}\right]$ ) generated by the components of $p$-Isog $\otimes E$ (resp. $p$-I $\left.\operatorname{sog}_{0}\right)$. We have $\mathbb{Q}\left[p\right.$-Isog $\left.{ }^{\mu}\right]=\mathbb{Q}[p$-Isog 0$]$ because $p$-Isog ${ }^{\mu}$ is

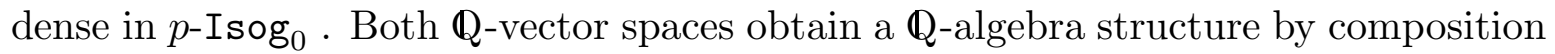
of $p$-isogenies. We define a commutative diagram of $\mathbb{Q}$-algebra homomorphisms

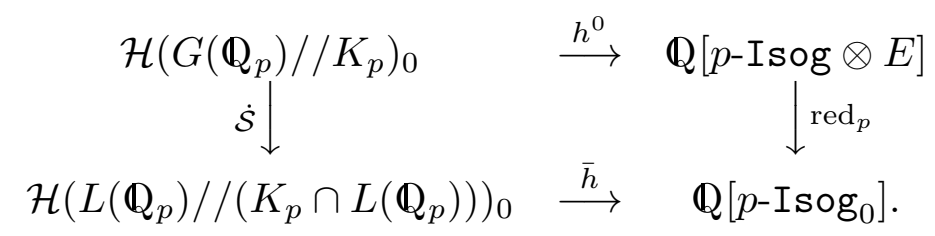

Here $L \subset G_{\mathbb{Q}_{p}}$ is the centralizer of the norm of the minuscule coweight $\mu$ of $G$ associated to $(G, X)$. The subscript ( $)_{0}$ in $\mathcal{H}\left(G\left(\mathbb{Q}_{p}\right) / / K_{p}\right)_{0}$ denotes the subalgebra of the Hecke algebra of functions with integral support such that if we invert the characteristic function of $K_{p}\left(p \cdot \mathrm{id}_{V}\right) K_{p}$ we get $\mathcal{H}\left(G\left(\mathbb{Q}_{p}\right) / / K_{p}\right)$, similar for $L$. We take $\mathbb{Q}$ as the coefficient ring for the Hecke algebras. The homomorphism $\dot{\mathcal{S}}$ is a twisted version of the Satake homomorphism already defined in [We2]. It is injective. On the right hand side $\operatorname{red}_{p}$ denotes the specialization map of cycles. Then elements in $\mathbb{Q}[p$-Isog $\otimes E]$ give rise to correspondences on $\operatorname{Sh}_{K}(G, X)$ and $h^{0}$ is simply the definition of Hecke correspondences on $\operatorname{Sh}_{K}(G, X)$. Finally, to construct $\bar{h}$ we define it as a homomorphism $\mathcal{H}\left(L\left(\mathbb{Q}_{p}\right) / /\left(K_{p} \cap L\left(\mathbb{Q}_{p}\right)\right) \longrightarrow \mathbb{Q}\left[p\right.\right.$-Isog $\left.{ }^{\mu}\right]$ and then use $\mathbb{Q}\left[p-\operatorname{Isog}^{\mu}\right]=\mathbb{Q}\left[p-\operatorname{Isog}_{0}\right]$.

Once we have the diagram $(*)$, the result follows from a group theoretic statement of elements in the Hecke algebra which has been proved by the first author in his 
thesis.

We will now give an overview on the structure of this article: In the first chapter we introduce notations and the second chapter introduces the Dieudonné modules with the additional structure which are considered in the sequel, the so-called unitary Dieudonné modules of signature $(n-1,1)$.

In the third chapter we give a precise description of the isomorphism classes of the reduction mod $p$ of the unitary Dieudonné modules of signature $(n-1,1)$ using a theorem of Moonen [Mo] which simplifies our previous arguments considerably (3.5). Further we calculate the dimension of the group of automorphisms of such a reduction (3.13) because this gives the codimension of the corresponding Ekedahl-Oort stratum by [We3].

The fourth chapter contains a criterion to recover the Newton polygon of a unitary Dieudonné module from its reduction $\bmod p(4.2)$. In particular, we see that the Oort stratification is finer than the Newton polygon stratification. Further in (4.9) we give the description of the homomorphism induced by $p$-isogenies induced on Dieudonné modules for non-supersingular points.

In the fifth chapter we compare Newton polygon, Ekedahl-Oort and final stratification in detail and obtain the dimension of the supersingular locus of $\operatorname{Sh}_{K}(G, X) \otimes$ $\kappa\left(E_{v}\right)$.

The content of the sixth chapter is the definition of the generic model for the moduli space of $p$-isogenies and the lower bound for the dimension of components of $p$-Isog $\otimes \kappa\left(E_{v}\right)(6.15)$.

In the seventh chapter we examine the source and target morphisms $p$-Isog $\otimes$ $\kappa\left(E_{v}\right) \longrightarrow \operatorname{Sh}_{K}(G, X) \otimes \kappa\left(E_{v}\right)$. We show that they are finite (7.3) over the nonsupersingular locus. In (7.5) we describe the deformation functors of $\mu$-ordinary $p$-isogenies which allows to show that the $\mu$-ordinary locus in $p$-Isog $\otimes \kappa\left(E_{v}\right)$ is dense if $n$ is even (7.8). It further implies that in this case every point of $p$-Isog $\otimes \kappa\left(E_{v}\right)$ can be lifted to characteristic zero (7.9) and that source and target are finite locally free over the generic stratum although this last fact is proved only in 8.7 due to reasons of notation.

The last chapter contains now the definition of $h^{0}$ and $\bar{h}$, statement and proof of the main theorem $(8.10-8.12)$.

We are grateful to U. Görtz and S. Orlik for helpful remarks on preliminary versions of the manuscript. 


\section{Notations}

(1.1) Let $p>2$ be a fixed prime. Let $\overline{\mathbb{Q}}_{p}$ be an algebraic closure of $\mathbb{Q}_{p}$ and fix an embedding $\overline{\mathbb{Q}} \hookrightarrow \overline{\mathbb{Q}}_{p}$ where $\overline{\mathbb{Q}}$ denotes the algebraic closure of $\mathbb{Q}$ in $\mathbb{C}$. Further, we fix a square root $\sqrt{-1}$ of -1 in $\mathbb{C}$.

(1.2) Throughout we denote by $\mathcal{I}=\left(B,{ }^{*}, V,\langle\rangle, G,, O_{B}, \Lambda, h, \mu\right)$ the collection of the following data:

- $B$ denotes a simple $\mathbb{Q}$-algebra such that $B \otimes_{\mathbb{Q}} \mathbb{R} \cong M_{m}(\mathbb{C})$ and such that $B \otimes_{\mathbb{Q}} \mathbb{Q}_{p} \cong M_{m}(K)$ where $K$ is a quadratic unramified extension of $\mathbb{Q}_{p}$.

- * is a positive involution on $B$.

- $V \neq(0)$ is a finitely generated left $B$-module.

- $\langle$,$\rangle is a nondegenerate skew-hermitian \mathbb{Q}$-valued form on $V$ (i.e. $\langle$,$\rangle is alternating$ and $\left\langle b v, v^{\prime}\right\rangle=\left\langle v, b^{*} v^{\prime}\right\rangle$ for all $b \in B$ and $\left.v, v^{\prime} \in V\right)$.

- $G$ denotes the algebraic $\mathbb{Q}$-group of $B$-linear symplectic similitudes of $(V,\langle\rangle$,$) .$

- $O_{B}$ is a ${ }^{*}$-invariant $\mathbb{Z}_{(p)}$-order such that $O_{B} \otimes \mathbb{Z}_{p}$ is a maximal order of $B_{\mathbb{Q}_{p}}$.

- $\Lambda$ denotes an $O_{B}$-invariant $\mathbb{Z}_{p}$-lattice of $V \otimes_{\mathbb{Q}} \mathbb{Q}_{p}$ such that the alternating form on $\Lambda$ which is induced from $\langle$,$\rangle is a perfect \mathbb{Z}_{p}$-form.

- $h$ is a homomorphism of real algebraic groups $h: \mathbf{S}=\operatorname{Res}_{\mathbb{C} / \mathbb{R}}\left(\mathbb{G}_{m, \mathbb{C}}\right) \longrightarrow G_{\mathbb{R}}$ such that $h$ defines a Hodge structure of type $\{(-1,0),(0,-1)\}$ on $V$ (with the sign convention of [De]) and such that

$$
V_{\mathbb{R}} \times V_{\mathbb{R}} \longrightarrow \mathbb{R}, \quad\left(v, v^{\prime}\right) \mapsto\left\langle v, h(\sqrt{-1}) v^{\prime}\right\rangle
$$

is symmetric and positive definite on $V_{\mathbb{R}}$.

- $\mu$ denotes the composition

$$
\mathbb{G}_{m, \mathbb{C}} \longrightarrow \mathbf{S}_{\mathbb{C}} \stackrel{h_{\mathbb{C}}}{\longrightarrow} G_{\mathbb{C}}
$$

where the first arrow is the embedding $\mathbb{G}_{m, \mathbb{C}} \hookrightarrow \mathbf{S}_{\mathbb{C}}=\prod_{\operatorname{Gal}(\mathbb{C} / \mathbb{R})} \mathbb{G}_{m, \mathbb{C}}$ whose image is the factor of $\mathbf{S}_{\mathbb{C}}$ corresponding to the identity in $\operatorname{Gal}(\mathbb{C} / \mathbb{R})$.

We can and we do fix an isomorphism $B \otimes \mathbb{Q}_{p} \cong M_{m}(K)$ such that $O_{B} \otimes \mathbb{Z}_{p}$ is identified with $M_{m}\left(O_{K}\right)$.

(1.3) The algebraic group $G$ over $\mathbb{Q}$ is reductive and connected. The assumption $B \otimes \mathbb{R} \cong M_{m}(\mathbb{C})$ implies that $G_{\mathbb{R}}$ is isomorphic to the group of unitary similitudes $G U(r, s)$ of an hermitian form of signature $(r, s)$ for nonnegative integers $r$ and $s$ with

$$
r+s=n:=\operatorname{dim}_{\mathbb{Q}}(V) / 2 m .
$$


Without loss of generality we can assume that $r \geq s$.

We denote by $\left(X^{*}, R, X_{*}, R^{\vee}, \Delta\right)$ the based root datum of $G$ with its action of $\Gamma=\operatorname{Gal}(\overline{\mathbb{Q}} / \mathbb{Q})$ and by $\Omega$ its Weyl group. Let $\{\mu\}$ be the $G(\mathbb{C})$-conjugacy class of $\mu$ which we consider as an element of $X_{*} / \Omega$. The group $\Gamma$ acts continuously on the discrete groups $X_{*}$ and $\Omega$ such that $\gamma(w \lambda)={ }^{\gamma} w \gamma(\lambda)$ for $\gamma \in \Gamma, w \in \Omega$ and $\lambda \in X_{*}$. Hence, we get an induced continuous action of $\Gamma$ on $X_{*} / \Omega$. The stabilizer of $\{\mu\}$ in $\Gamma$ is open and defines a finite extension $E$ of $\mathbb{Q}$ which is called the reflex field of $\mathcal{I}$. It is easy to see (e.g. [We1] 2.3.2) that $E=\mathbb{Q}$ if $r=s$ and that $E$ is a quadratic imaginary extension otherwise.

Via the chosen embedding $\overline{\mathbb{Q}} \hookrightarrow \overline{\mathbb{Q}}_{p}$ we can consider $\{\mu\}$ as a $G\left(\overline{\mathbb{Q}}_{p}\right)$-conjugacy class of cocharacters. Denote by $v \mid p$ the place of $E$ given by the chosen embedding $\overline{\mathbb{Q}} \hookrightarrow \overline{\mathbb{Q}}_{p}$. Then the field of definition of $\{\mu\}$ with respect to the $\operatorname{Gal}\left(\overline{\mathbb{Q}}_{p} / \mathbb{Q}_{p}\right)$-action is the $v$-adic completion of $E$. Let $\kappa$ be the residue class field of $v$. The action of $\operatorname{Gal}\left(\overline{\mathbb{Q}}_{p} / \mathbb{Q}_{p}\right)$ factors over $\operatorname{Gal}\left(K / \mathbb{Q}_{p}\right)$. We denote by $\sigma$ the nontrivial element in $\operatorname{Gal}\left(K / \mathbb{Q}_{p}\right)$.

Note that the assumption on $B_{\mathbb{Q}_{p}}$ and the existence of $\Lambda$ imply that $G_{\mathbb{Q}_{p}}$ is unramified over $\mathbb{Q}_{p}$. More precisely, the stabilizer of $\Lambda$ defines a reductive model $\tilde{G}$ of $G_{\mathbb{Q}_{p}}$ over $\mathbb{Z}_{p}$.

(1.4) Denote by $\mathbf{A}_{f}^{p}$ the ring of finite adeles of $\mathbb{Q}$ with trivial $p$-th component. We fix an open compact subgroup $K^{p} \subset G\left(\mathbf{A}_{f}^{p}\right)$ and denote by $\mathcal{M}=\mathcal{M}_{\mathcal{I}, K^{p}}$ the associated moduli space, defined by Kottwitz [Ko]. We assume $K^{p}$ to be sufficiently small, such that the moduli problem is representable by a scheme. This scheme is then smooth and quasi-projective over the localization of $O_{E}$ in $p$. It classifies tuples $(A, \iota, \lambda, \bar{\eta})$ where $A$ is an abelian scheme up to prime-to- $p$-isogeny, $\lambda$ is a $\mathbb{Q}$-homogeneous polarization of $A$ of degree prime to $p, \iota: O_{B} \rightarrow \operatorname{End}(A) \otimes_{\mathbb{Z}} \mathbb{Z}_{(p)}$ is an involution preserving $\mathbb{Z}_{(p)^{-}}$ algebra homomorphism where the involution is ${ }^{*}$ on $O_{B}$ and the Rosati-Involution given by $\lambda$ on $\operatorname{End}(A) \otimes_{\mathbb{Z}} \mathbb{Z}_{(p)}$, and where $\bar{\eta}$ is a $K^{p}$-level structure. Further $(A, \iota, \lambda, \bar{\eta})$ satisfies the determinant condition, i.e. we have an identity of polynomial functions on $O_{B}$

$$
\operatorname{det}(b \mid \operatorname{Lie}(A))=\operatorname{det}\left(b \mid V_{0}\right)
$$

where $V_{0} \subset V_{E_{v}}$ is the weight zero space of some $\mu \in\{\mu\}$ (see [Ko2] $\S 5$ or [RZ] 3.23 a) for a precise formulation of the determinant condition).

It follows from the general theory of Shimura varieties that $\mathcal{M}_{\mathcal{I}, K^{p}}$ has relative 
dimension

$$
2\langle\rho,\{\mu\}\rangle=r s
$$

where $\rho$ denotes the halfsum of positive roots in the based root datum of $G$.

(1.5) We choose in the conjugacy class $\{\mu\}$ the unique element $\mu$ which is dominant. As $G_{E_{v}}$ is unramified over $E_{v}$, it is already defined over $O_{E_{v}}$. Set

$$
N(\mu)=\sum_{\gamma \in \operatorname{Gal}\left(E_{v} / \mathbb{Q}_{p}\right)} \gamma(\mu) .
$$

This a one-parameter subgroup of $G_{\mathbb{Q}_{p}}$ which extends (uniquely) to a homomorphism $\mathbb{G}_{m, \mathbb{Z}_{p}} \longrightarrow \tilde{G}$. We denote by $L \subset G_{\mathbb{Q}_{p}}$ the centralizer of $N(\mu)$ in $G_{\mathbb{Q}_{p}}$. This is a Levi subgroup of $G_{\mathbb{Q}_{p}}$ which is unramified as reductive group over $\mathbb{Q}_{p}$.

(1.6) Denote by $K_{p}$ the stabilizer of $\Lambda$ in $G\left(\mathbb{Q}_{p}\right)$ with the notations of $(1.2)$, i. e. $K_{p}=\tilde{G}\left(\mathbb{Z}_{p}\right)$. Let $\mathcal{H}_{\mathbb{Q}}(G)=\mathcal{H}_{\mathbb{Q}}\left(G\left(\mathbb{Q}_{p}\right) / / K_{p}\right)$ be the Hecke algebra of $\mathbb{Q}$-valued locally constant $K_{p}$-biinvariant functions on $G\left(\mathbb{Q}_{p}\right)$. It is commutative as $K_{p}$ is a hyperspecial subgroup of $G\left(\mathbb{Q}_{p}\right)$. The Cartan decomposition of $G\left(\mathbb{Q}_{p}\right)$ shows that we have an identification

$$
K_{p} \backslash G\left(\mathbb{Q}_{p}\right) / K_{p}=\left(X_{*} / \Omega\right)^{\sigma}
$$

where $\sigma \in \operatorname{Gal}\left(K / \mathbb{Q}_{p}\right)$ denotes the nontrivial element.

We denote by $\mathcal{H}_{\mathbb{Q}}(G)_{0}$ the subalgebra of $\mathcal{H}_{\mathbb{Q}}(G)$ which consists of functions whose support lies in $\operatorname{End}_{\mathbb{Z}_{p}}(\Lambda)$. If $P$ is the double coset of $p \operatorname{id}_{V}$, we have $\mathcal{H}_{\mathbb{Q}}(G)_{0}[1 / P]=$ $\mathcal{H}_{\mathbb{Q}}(G)$ as $p \operatorname{id}_{V}$ is central in $G\left(\mathbb{Q}_{p}\right)$.

We denote by $H=H_{G,\{\mu\}} \in \mathcal{H}_{\mathbb{Q}}(G)_{0}$ the Hecke polynomial associated to $G$ and the conjugacy class $\{\mu\}$ (see e.g. [We2] $\S 2$ for the definition and the fact that its coefficients lie in $\left.\mathcal{H}_{\mathbb{Q}}(G)_{0}\right)$.

Further ${ }_{L} K=L\left(\mathbb{Q}_{p}\right) \cap K_{p}$ is a hyperspecial subgroup of $M\left(\mathbb{Q}_{p}\right)$. It is also equal to the centralizer of the extension of $N(\mu)$ in $\tilde{G}\left(\mathbb{Z}_{p}\right)$. Again we get Hecke algebras $\mathcal{H}_{\mathbb{Q}}(L)=\mathcal{H}_{\mathbb{Q}}\left(L\left(\mathbb{Q}_{p}\right) / /{ }_{L} K\right)$ and $\mathcal{H}_{\mathbb{Q}}(L)_{0}$.

(1.7) Let $S$ be an $O_{E, v}$-scheme and let $\left(A_{1}, \iota_{1}, \lambda_{1}, \bar{\eta}_{1}\right),\left(A_{2}, \iota_{2}, \lambda_{2}, \bar{\eta}_{2}\right) \in \mathcal{M}(S)$ be two $S$-valued points. A p-isogeny

$$
f:\left(A_{1}, \iota_{1}, \lambda_{1}, \bar{\eta}_{1}\right) \longrightarrow\left(A_{2}, \iota_{2}, \lambda_{2}, \bar{\eta}_{2}\right)
$$


is an $O_{B}$-linear isogeny $f: A_{1} \longrightarrow A_{2}$ such that there exists an integer $c \geq 0$ with $(\alpha f)^{*} \lambda_{2}=p^{c} \lambda_{1}$.

The integer $c=c(f)$ is called the multiplicator of $f$.

We have the obvious notion of the pullback of a $p$-isogeny with respect to a morphism of schemes $S^{\prime} \longrightarrow S$ and the notion of an isomorphism of two $p$-isogenies. We get a functor $p$-Isog $=p$-Isog ${\operatorname{II~}, K^{p}}$ on the category of $O_{E, \nu}$-schemes. The functor $p$-Isog ${ }^{c}$ of isomorphism classes of $p$-isogenies with multiplicator $c$ is an open and closed subfunctor.

An important example of a $p$-isogeny is the relative Frobenius

$$
\text { Fr: } A \longrightarrow A^{(q)}
$$

where $q$ denotes the number of elements in $\kappa\left(E_{v}\right)$, i.e. $q=p$ if $r=s$ and $q=p^{2}$ otherwise. Its multiplicator is $\log _{p} q$.

Sending a $p$-isogeny to its source (resp. its target) defines morphisms

$$
s: p-\operatorname{Isog}^{c} \longrightarrow \mathcal{M}_{\mathcal{I}, K^{p}}, \quad t: p-\operatorname{Isog}^{c} \longrightarrow \mathcal{M}_{\mathcal{I}, K^{p}}
$$

which are representable by projective surjective morphisms. In particular $p$-Isog ${ }^{c}$ (and hence $p$-Isog) is representable by a scheme. Further, the morphisms are finite étale in characteristic zero. Hence $p$-Isog $\otimes E$ is smooth.

For every $p$-isogeny $f$ we denote by $f^{\vee}$ its dual. It is again a $p$-isogeny of the same multiplicator as $f$.

\section{Unitary p-divisible groups}

(2.1) Let $S$ be an $O_{E_{v}}$-scheme which is $p$-adically complete. A $p$-divisible $\mathcal{I}$-module over $S$ is a triple $(X, \iota, \lambda)$ where $X$ is a $p$-divisible group over $S, \iota: O_{K} \longrightarrow \operatorname{End}(X)$ is a ring homomorphism and where $\lambda:(X, \iota) \stackrel{\sim}{\longrightarrow}\left(X^{\vee}, \iota^{\vee}\right)$ is an $O_{K}$-linear isomorphism of $p$-divisible groups such that $\lambda^{\vee}=-\lambda$. Here ()$^{\vee}$ denotes the Serre dual and by $\iota^{\vee}: O_{K} \longrightarrow \operatorname{End}\left(X^{\vee}\right)$ we mean the homomorphism $\iota^{\vee}(a)=\iota\left(a^{*}\right)^{\vee}$ for $a \in O_{K}$. We further require that we have for all $n \geq 0$ an identity of polynomial functions on $O_{K}$

$$
\operatorname{det}\left(\iota(a) \mid \operatorname{Lie}\left(X \times_{S} S_{n}\right)\right)=\operatorname{det}\left(a \mid V_{0} \otimes_{M_{m}(K)} K^{m}\right)
$$

where $S_{n}=\operatorname{Spec}\left(\mathcal{O}_{S} / p^{n+1} \mathcal{O}_{S}\right)$. As $X \times_{S} S_{n}$ is formally smooth over $S_{n}, \operatorname{Lie}\left(X \times_{S} S_{n}\right)$ is a finite locally free $\mathcal{O}_{S_{n}}$-module and (2.1.1) holds for all $n \geq 0$ if it holds for one $n \geq 0$. 
We want to reinterprete this condition further. We fix the isomorphism

$$
O_{K} \otimes_{\mathbb{Z}_{p}} O_{K} \stackrel{\sim}{\longrightarrow} O_{K} \times O_{K}, \quad a \otimes a^{\prime} \mapsto\left(a a^{\prime}, a^{*} a^{\prime}\right)
$$

As the determinant condition (2.1.1) can be checked after some finite étale base change $S^{\prime} \longrightarrow S$ we can assume that $S$ is also an $O_{K}$-scheme. We obtain a decomposition of the $O_{K} \otimes \mathcal{O}_{S}$-module $\operatorname{Lie}(X)$ of the form $\operatorname{Lie}(X)=L_{0} \oplus L_{1}$ where $L_{i}$ is an $\mathcal{O}_{S^{-}}$ submodule. The determinant condition is then equivalent to the condition

$$
\operatorname{rk}_{\mathcal{O}_{S}}\left(L_{0}\right)=r, \quad \operatorname{rk}_{\mathcal{O}_{S}}\left(L_{1}\right)=s
$$

where $r$ and $s$ are the integers defined in (1.3).

(2.2) Let $S$ be as above and let $(A, \iota, \lambda, \bar{\eta})$ be an $S$-valued point of $\mathcal{M}_{\mathcal{I}, K^{p}}$. The $p$-primary torsion of $(A, \iota, \lambda)$ defines a $p$-divisible $O_{B} \otimes \mathbb{Z}_{p}$-module $\left(X^{\prime}, \iota^{\prime}\right)$ together with an $O_{B}$-linear isomorphism $\lambda^{\prime}:\left(X^{\prime}, \iota^{\prime}\right) \stackrel{\sim}{\longrightarrow}\left(X^{\prime \vee}, \iota^{\prime \vee}\right)$. As $O_{B} \otimes \mathbb{Z}_{p}=M_{m}\left(O_{K}\right)$ we can apply Morita equivalence and we get a $p$-divisible $\mathcal{I}$-module over $S$ which we call associated to $(A, \iota, \lambda, \bar{\eta})$.

(2.3) We are going to study the category of $p$-divisible $\mathcal{I}$-modules over a fixed algebraically closed field $k$ of characteristic $p$ which is endowed with an $O_{K}$-algebra structure. Denote by $W(k)$ its ring of Witt vectors. The Frobenius automorphism of $W(k)$ is denoted by $\sigma$. It is a lifting of the usual Frobenius Frob $k$ of $k$ which sends $x$ to $x^{p}$. Our main tool will be Dieudonné theory.

(2.4) A Dieudonné module over $k$ is a finitely generated free $W(k)$-module $M$ together with a $\sigma$-linear endomorphism $F$ and a $\sigma^{-1}$-linear endomorphism $V$ of $M$ such that $F V=V F=p \cdot \mathrm{id}_{M}$. A Dieudonné space over $k$ is a finite-dimensional $k$-vector space $\bar{M}$ together with a Frob ${ }_{k}$-linear endomorphism $F$ and a Frob ${ }_{k}^{-1}$-linear endomorphism $V$ such that $F V=V F=0$. If $(M, F, V)$ is a Dieudonné module then its reduction modulo $p$ is a Dieudonné space.

A graded Dieudonné module over $k$ is a tuple $\left((M, F, V), M=M_{0} \oplus M_{1}\right)$ where $(M, F, V)$ is a Dieudonné module over $k$ and where $M=\bigoplus_{i \in \mathbb{Z} / 2 \mathbb{Z}} M_{i}$ is a decomposition of $W(k)$-submodules such that $F$ and $V$ are homogeneous of degree 1 . We have the analogous definition of a graded Dieudonné space over $k$. If $((\bar{M}, F, V), \bar{M}=$ $\left.\bar{M}_{0} \oplus \bar{M}_{1}\right)$ is a graded Dieudonné space we call the pair

$$
\left(\operatorname{dim}_{k}\left(\bar{M}_{0} / V \bar{M}_{1}\right), \operatorname{dim}_{k}\left(\bar{M}_{1} / V \bar{M}_{0}\right)\right)
$$


its signature and denote it by $\operatorname{sign}(\bar{M})$. The signature of a graded Dieudonné module is defined as the signature of its reduction modulo $p$. We have the obvious notion of a morphism of graded Dieudonné modules (resp. of graded Dieudonné spaces). Finally by inverting $p$ we get the notion of a graded isocrystal.

A quasi-unitary Dieudonné module over $k$ is a tuple $\underline{M}=((M, F, V),\langle\rangle,, M=$ $\left.M_{0} \oplus M_{1}\right)$ such that $\left((M, F, V), M=M_{0} \oplus M_{1}\right)$ is a graded Dieudonné module over $k$ and $\langle\rangle:, M \times M \rightarrow W(k) \otimes_{\mathbb{Z}} \mathbb{Q}$ is a non-degenerate alternating pairing such that

$$
\langle F x, y\rangle=\langle x, V y\rangle^{\sigma}
$$

and such that $M_{0}$ and $M_{1}$ are totally isotropic with respect $\langle$,$\rangle . We call \underline{M}$ a unitary Dieudonné module if $\langle$,$\rangle is W(k)$-valued and perfect. We have further the obvious notions of a unitary Dieudonné space over $k$ and a unitary isocrystal over $k$.

(2.5) By (covariant) Dieudonné theory we have an equivalence between the category of unitary Dieudonné modules $\left((M, F, V),\langle\rangle,, M=M_{0} \oplus M_{1}\right)$ over $k$ of signature $(r, s)$ and the category of $p$-divisible $\mathcal{I}$-modules $(X, \iota, \lambda)$ over $k$. Via this equivalence we have a canonical identification of $\operatorname{Lie}(X)$ with $M / V M$ such that $L_{0}=M_{0} / V M_{1}$ and $L_{1}=M_{1} / V M_{0}$ where $\operatorname{Lie}(X)=L_{0} \oplus L_{1}$ is the decomposition of (2.1).

(2.6) Let $\underline{M}_{1}$ and $\underline{M}_{2}$ be two graded Dieudonné spaces over $k$. For every $k$-algebra $R$ we denote by $\underline{\operatorname{Hom}}_{\mathrm{gD}}\left(\underline{\bar{M}}_{1}, \underline{\bar{M}}_{2}\right)(R)$ the set of $R$-linear maps $\varphi: M_{1} \otimes_{k} R \rightarrow M_{2} \otimes_{k} R$ which preserve the grading and such that the diagrams

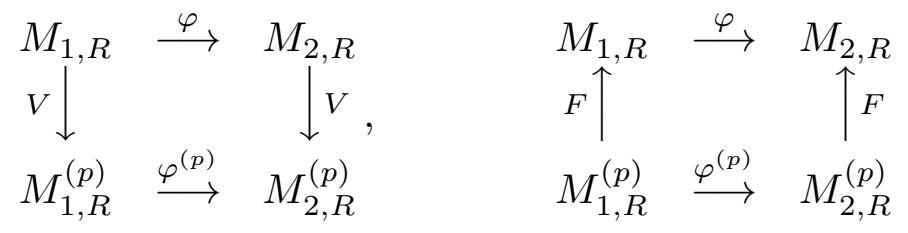

commute. This defines a functor which is representable by a closed subscheme of $\underline{\operatorname{Hom}}_{k}\left(M_{1}, M_{2}\right)$. In particular it is affine and of finite type over $k$. If we consider for $\underline{M}_{1}=\underline{M}_{2}=\underline{\bar{M}}$ only those $\varphi$ which are invertible, we get the algebraic group scheme $\underline{\mathrm{Aut}}_{\mathrm{gD}}(\underline{\bar{M}})$ which is affine as a closed subgroup scheme of $G L(M)$.

If $\underline{\bar{M}}$ is a unitary Dieudonné space over $k$ we denote by $\underline{\operatorname{Aut}}_{\mathrm{uD}}(\underline{\bar{M}})$ the closed subgroup scheme of automorphisms which also preserve the symplectic form.

\section{Unitary Dieudonné spaces of signature $(n-1,1)$}


(3.1) From now on $\underline{M}=\left((M, F, V), M=M_{0} \oplus M_{1},\langle\rangle,\right)$ denotes a unitary Dieudonné module over an algebraically closed field $k$ of characteristic $p$ and we denote by $\underline{\bar{M}}$ the unitary Dieudonné space obtained from $\underline{M}$ by reduction modulo $p$. We assume that the signature of $\underline{M}$ is $(n-1,1)$

Let $N=\left(M_{\mathbb{Q}}, F \otimes \mathrm{id}\right)$ be its isocrystal. For $\lambda \in \mathbb{Q}$ denote by $N_{\lambda}$ "the" simple isocrystal of slope $\lambda$. Then

$$
N \cong N(r) \oplus\left(N_{1 / 2}\right)^{n-2 r}
$$

for some integer $0 \leq r \leq n / 2$ where

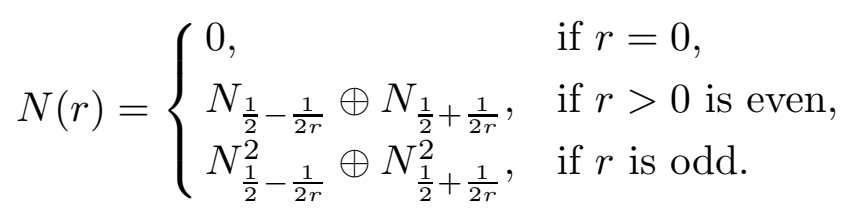

Further the decomposition $N(r) \oplus\left(N_{\frac{1}{2}}\right)^{n-2 r}$ is a decomposition of unitary isocrystals, i.e. it is orthogonal and graded.

It follows that the isocrystal of a unitary Dieudonné module of signature $(n-1,1)$ is determined by its first Newton slope. If we denote by $p_{r}$ the Newton-polygon associated to $N(r) \oplus\left(N_{1 / 2}\right)^{n-2 r}$, we have $p_{0} \leq p_{1} \leq \ldots \leq p_{[n / 2]}$ where we write $p \leq q$ if $p$ lies above $q$.

(3.2) The following two examples will be of vital importance:

(1) We define a superspecial unitary Dieudonné module $\underline{S}$ as follows: As $W(k)$ module it is a module of rank 2 generated by elements $g$ and $h$. We set $M_{0}=W(k) g$ and $M_{1}=W(k) h$. The alternating form is given by $\langle g, h\rangle=1$, and we have $F g=-h$ and $V g=h$. Then $S$ is a unitary Dieudonné module of signature $(1,0)$. We also write $\underline{\bar{S}}$ for its reduction $\bmod p$ and $S$ for the underlying Dieudonné module of $\underline{S}$.

(2) For an integer $n \geq 1$ define a unitary Dieudonné module $\underline{B}(n)$ as follows: As $W(k)$-module it has a basis $\left(e_{1}, \ldots, e_{n}, f_{1}, \ldots, f_{n}\right)$ and the grading is given by $M_{0}=W(k) e_{1} \oplus \ldots \oplus W(k) e_{n}$ and $M_{1}=W(k) f_{1} \oplus \ldots \oplus W(k) f_{n}$. The alternating form is defined by

$$
\left\langle e_{i}, f_{j}\right\rangle=(-1)^{i-1} \delta_{i j} .
$$

Finally $F$ and $V$ are given by

$$
V e_{i}=f_{i+1}, \quad \text { for } i=1, \ldots, n-1,
$$




$$
\begin{aligned}
V f_{n} & =e_{1}, \\
F e_{i} & =f_{i-1}, \\
F f_{1} & =(-1)^{n} e_{n} .
\end{aligned} \quad \text { for } i=2, \ldots, n,
$$

This is a unitary Dieudonné module of signature $(n-1,1)$ which we call braid of length $n$. We also write $\underline{\bar{B}}(n)$ for its reduction modulo $p$ and denote the images of $e_{i}$ and $f_{i}$ again by $e_{i}$ resp. $f_{i}$.

(3.3) Lemma: The isocrystal associated to $\underline{B}(n)$ has the following Newton slopes:

(1) If $n$ is odd, every Newton slope is $1 / 2$.

(2) If $n$ is even, the Newton slopes are $\frac{n / 2-1}{n}$ and $\frac{n / 2+1}{n}$. In particular, it has only slopes 0 and 1 if and only if $n=2$.

Proof: Denote by $\lambda(n)$ the first Newton slope of $\underline{B}(n)$. By [Zi1] $\S 6.12$ we have

$$
\lambda(n)=\lim _{n \rightarrow \infty} \frac{1}{n} \max \left\{k \in \mathbb{Z} \mid V^{n} M \subset p^{k} M\right\}
$$

and hence $\lambda(n)=1 / 2$ if $n$ is odd and $\lambda(n)=\frac{n / 2-1}{n}$ if $n$ is even. Therefore the lemma follows from (3.1).

(3.4) Lemma: Assume that the signature of $\underline{M}$ is either $(r, 0)$ or $(0, r)$ for some $r \geq 1$. Then $\underline{M}$ is superspecial, i.e. $F M=V M$.

Proof: Assume that the signature is $(r, 0)$ (the other case is analogous). This means that $V M_{0}=M_{1}$. This implies $F^{-1} M_{0}^{\perp}=M_{1}^{\perp}$ and as we have $M_{i}^{\perp}=M_{i+1}$ we get $F M_{0}=M_{1}$. It follows that $F M_{1}=V M_{1}=p M_{0}$.

(3.5) Proposition: Let $\underline{\bar{M}}$ be a unitary Dieudonné space over $k$ of signature $(n-1,1)$. Then $\underline{\bar{M}}$ is isomorphic to

$$
\underline{\bar{B}}(r) \oplus \underline{\bar{S}}^{n-r}
$$

for some integer $r$ with $1 \leq r \leq n$.

Proof: This follows easily from a theorem of Moonen [Mo]: He has shown (in much greater generality) that the number of isomorphism classes of unitary Dieudonné spaces of signature $(n-1,1)$ is given by the number of elements in the quotient of the Weyl group $\Omega$ of $G$ by the Weyl group $\Omega_{\mu}$ of the centralizer of $\mu$ in $G_{\mathbb{C}}$. Now $\Omega$ equals the symmetric group $S_{n}$ and $\Omega_{\mu}$ can be identified with $S_{n-1} \times S_{1}$, embedded in the obvious way in $S_{n}$. Hence we see that there are precisely $n$ of such isomorphism 
classes. As the unitary Dieudonné spaces $\underline{\bar{B}}(r) \oplus \underline{\bar{S}}^{n-r}$ are pairwise nonisomorphic for different $r$, the claim follows.

(3.6) In order to study the stratification of the special fibre of $\mathcal{M}_{\mathcal{I}, K^{p}}$ by isomorphism types of unitary Dieudonné spaces (the Oort stratification in the terminology of [We3]) we have to compute the dimension of the group of automorphisms of a unitary Dieudonné space. This will be done in the next sections. We refer to [We3] for the definition of a Dieudonné space over an arbitrary $k$-algebra $R$. We have the obvious definition of $\underline{\bar{B}}(n)$ and $\underline{\bar{S}}$ over $R$.

(3.7) Let $\underline{\bar{B}}(n)=\left(\bar{B}(n), F, V,\langle\rangle,, \bar{B}(n)=\bar{B}(n)_{0} \oplus \bar{B}(n)_{1}\right)$ be a braid of length $n$. We use the notations of $(3.2)(2)$ and denote the image of $e_{i}$ resp. $f_{i}$ in $\bar{B}(n)$ again by $e_{i}$ resp. $f_{i}$. For $r=0, \ldots,[n / 2]$ we set

$$
M^{r}=\bigoplus_{i=0}^{[(n-1) / 2]} k e_{n-2 i} \oplus \bigoplus_{i=r}^{[n / 2]-1} k e_{n-1-2 i}
$$

We can make this definition over arbitrary $k$-algebras. If we write $V^{*} W=V^{-1} W \cap$ $\bar{B}(n)_{i+1}$ for a subspace $W$ of $\bar{B}(n)_{i}$, we have

$$
M^{r}=\left(V^{*} F\right)^{r} \bar{B}(n)_{0} .
$$

Further, we set

$$
t= \begin{cases}n / 2, & \text { if } n \text { is even, } \\ (n-3) / 2, & \text { if } n \text { is odd. }\end{cases}
$$

(3.8) Let $\varphi$ be an automorphism of $\underline{\bar{B}}(n)=\left(\bar{B}(n), F, V,\langle\rangle,, \bar{B}(n)=\bar{B}(n)_{0} \oplus \bar{B}(n)_{1}\right)$ over a $k$-algebra $R$. We claim that $\varphi$ is uniquely determined by $\varphi\left(e_{1}\right)$ and $\varphi\left(f_{1}\right)$ : It is clear that if we know $\varphi\left(e_{1}\right), \ldots, \varphi\left(e_{i}\right)$ and $\varphi\left(f_{1}\right), \ldots, \varphi\left(f_{i}\right)$ for $i<n$ we have $\varphi\left(f_{i+1}\right)=$ $V \varphi\left(e_{i}\right)$. Further $\varphi\left(e_{i+1}\right)$ has to satisfy $F\left(\varphi\left(e_{i+1}\right)\right)=\varphi\left(f_{i}\right)$ and $\left\langle\varphi\left(e_{i+1}\right), \varphi\left(f_{1}\right)\right\rangle=0$ and these conditions determine $\varphi\left(e_{i+1}\right)$ uniquely as $\varphi\left(e_{i+1}\right) \in \bar{B}(n)_{0}$.

As $\varphi\left(V \bar{B}(n)_{1}\right) \subset V \bar{B}(n)_{1}$, we have $\varphi\left(e_{1}\right)=\alpha_{\varphi} e_{1}$ for a unit $\alpha_{\varphi}$. This defines a homomorphism $\alpha: \underline{\operatorname{Aut}}(\underline{\bar{B}}(n)) \longrightarrow \mathbb{G}_{m}$. Set $r=p^{n}-1$ if $n$ is even, and $r=p^{n}+1$ if $n$ is odd. It is easy to see that the image of $\alpha$ is $\mu_{r}$, the scheme of $r$-th roots of unity.

Further, $\varphi\left(f_{1}\right) \in F M^{t}$ as $f_{1} \in F M^{t}$. As $\left\langle e_{1}, f_{i}\right\rangle=\delta_{1 i}$, there exists a unique $m_{\varphi} \in F\left(M^{t}\right)^{(p)}$ such that $\varphi\left(f_{1}\right)=\alpha_{\varphi}^{-1}\left(f_{1}+m_{\varphi}\right)$ and $\left\langle e_{1}, m_{\varphi}\right\rangle=0$, and this means that $m_{\varphi}$ is in the free submodule generated by $f_{2}, f_{4}, \ldots, f_{n-1}$ if $n$ is odd and in the 
submodule generated by $f_{3}, f_{5}, \ldots, f_{n-1}$ if $n$ is even. Denote this free submodule by $N$. We consider $N$ as $k$-scheme isomorphic to an affine space. From the construction above of $\varphi\left(e_{i}\right)$ and $\varphi\left(f_{i}\right)$ from $\varphi\left(e_{1}\right)$ and $\varphi\left(f_{1}\right)$ it follows that the morphism of schemes

$$
m: \underline{\operatorname{Aut}}(\underline{\bar{B}}(n)) \mapsto N, \quad \varphi \mapsto m_{\varphi}
$$

is an isomorphism of schemes (but not of group schemes). Hence we see:

Proposition: Set $r=p^{n}-1$ if $n$ is even and $r=p^{n}+1$ if $n$ is odd. Then the map

$$
\underline{\operatorname{Aut}}(\underline{\bar{B}}(n)) \longrightarrow \mu_{r}, \quad \varphi \mapsto \alpha_{\varphi}
$$

provides a surjective homomorphism of algebraic groups. Its kernel is the identity component $\underline{\operatorname{Aut}}(\underline{\bar{B}}(n))^{0}$. The morphism

$$
m: \operatorname{Aut}(\underline{\bar{B}}(n))^{0} \longrightarrow N
$$

is an isomorphism of varieties.

(3.9) Corollary: We have

$$
\operatorname{dim}(\underline{\operatorname{Aut}}(\underline{\bar{B}}(n)))= \begin{cases}\frac{n}{2}-1, & \text { if } n \text { is even, } \\ \frac{n-1}{2}, & \text { if } n \text { is odd. }\end{cases}
$$

(3.10) Proposition: Let $r$ and $m$ be positive integers.

(1) The group scheme $\underline{\mathrm{Aut}}_{\mathrm{uD}}\left(\underline{\bar{S}}^{m}\right)$ is constant with underlying group equal to the $\mathbb{F}_{p}$-valued points of the unitary group in $m$ variables (regarded as an abstract group).

(2) The scheme $\underline{\operatorname{Hom}}_{\mathrm{gD}}\left(\underline{\bar{S}}^{m}, \underline{\bar{B}}(r)\right)$ is isomorphic to the $m$-dimensional affine space if $r$ is odd and to $\alpha_{p^{r}}^{m}$ if $r$ is even.

Proof: Write $\underline{\bar{S}}^{m}=\underline{\bar{S}}_{1} \oplus \ldots \oplus \underline{\bar{S}}_{m}$ with $\underline{\bar{S}}_{i}=\underline{\bar{S}}$ and for each $1 \geq i \geq m$ choose a basis $\left(g_{i}, h_{i}\right)$ as in $(3.2)(1)$. Let $R$ be the coordinate ring of the affine scheme $\underline{\text { Aut }}_{\mathrm{uD}}\left(\underline{\bar{S}}^{m}\right)$. Let also $\Phi \in \underline{\mathrm{Aut}}_{\mathrm{uD}}\left(\underline{\bar{S}}^{m}\right)(R)$ be the universal automorphism. It preserves the grading, so write $\Phi\left(g_{j}\right)=\sum_{i} a_{i j} g_{i}$ and $\Phi\left(h_{j}\right)=\sum_{i} b_{i j} h_{i}$ and denote by $A$ and $B$ the matrices $\left(a_{i j}\right)$ and $\left(b_{i j}\right)$. As $\Phi$ commutes with $F$ and $V$, the matrices $A$ and $B$ satisfy

$$
A^{(p)}=B, \quad B^{(p)}=A
$$


and in particular $A=A^{\left(p^{2}\right)}$, i.e. $a_{i j} \in \mathbb{F}_{p^{2}}$. Moreover, as $\Phi$ preserves the form, one has

$$
\sum_{k=1}^{m} a_{k i} b_{k j}=\delta_{i j}
$$

or equivalently ${ }^{t} A B=1$. Therefore $R$ is the quotient of the polynomial ring $k\left[a_{i j}, b_{i j}\right]$ by the ideal given by the relations $(\mathrm{A})$ and ${ }^{t} A B=1_{m}$ which proves (1).

Choose a basis $\left(e_{1}, \ldots, e_{r}, f_{1}, \ldots, f_{r}\right)$ as in $(3.2)(1)$ for $\underline{\bar{B}}(r)$. To prove (2) we consider the coordinate ring $R$ of $\underline{\operatorname{Hom}}_{\mathrm{gD}}\left(\underline{\bar{S}}^{m}, \underline{\bar{B}}(r)\right)$ together with the universal homomorphism $\Phi \in \underline{\operatorname{Hom}}_{\mathrm{gD}}\left(\underline{\bar{S}}^{m}, \underline{\bar{B}}(r)\right)(R)$ sending $g_{j}$ to $\sum_{i} s_{i j} e_{i}$ and $h_{j}$ to $\sum_{i} t_{i j} f_{i}$. The fact that $\Phi$ commutes with $F$ and $V$ is equivalent to the equations

$$
\begin{gathered}
t_{1,1}^{p}=\cdots=t_{1, m}^{p}=0, \\
t_{r, 1}=\cdots=t_{r, m}=0, \\
s_{i+1, j}^{p}=-t_{i, j} \text { and } t_{i+1, j}^{p}=s_{i j}
\end{gathered}
$$

for $1 \leq i \leq r-1$ and $1 \leq j \leq m$. The equations (C) and (D) mean that the only nonzero $s_{i j}$, (resp. $t_{i j}$ ) have $i \equiv r(2)$ (resp. $i \equiv r-1(2)$ ) and, moreover, these are completely determined by $s_{r, j}$, as $(-1)^{\frac{r-i}{2}} s_{r, j}^{p^{r-i}}=s_{i, j}$ if $i \equiv r(2)$, and $(-1)^{\frac{r-i+1}{2}} s_{r, j}^{p^{r-i}}=t_{i, j}$ if $i \equiv r-1(2)$.

We have not yet used equation (B), which is non-redundant only if $1 \equiv r-1(2)$, i.e. if $r$ is even. In this case it forces $s_{r, j}$ to satisfy $s_{r, j}^{p^{r}}=\left((-1)^{\frac{r}{2}} t_{1, j}\right)^{p}=0$. Therefore

$$
R \cong k\left[s_{r, 1}, \ldots, s_{r, m}\right]
$$

if $r$ is odd and

$$
R \cong k\left[s_{r, 1}, \ldots, s_{r, m}\right] /\left(s_{r, 1}^{p^{r}}, \ldots, s_{r, m}^{p^{r}}\right),
$$

if $r$ is even.

(3.11) We keep the notations. The proof of $(3.10)(2)$ shows that we have

$$
\underline{\operatorname{Hom}}_{\mathrm{gD}}\left(\underline{\bar{S}}^{m}, \underline{\bar{B}}(r)\right)=\underline{\operatorname{Hom}}_{\mathrm{gD}}\left(\underline{\bar{S}}^{m}, \underline{\bar{B}}^{+}(r)\right)
$$

where we denote by $\underline{B}^{+}(r)$ the graded Dieudonné subspace which is generated by

$$
e_{r}, e_{r-2}, \ldots, e_{r \bmod 2}, f_{r-1}, f_{r-3}, \ldots, f_{(r-1) \bmod 2} .
$$

By definition, $\underline{\bar{B}}^{+}(r)$ is a totally isotropic subspace of $\underline{\bar{B}}(r)$ which is maximal totally isotropic because of its dimension. 
(3.12) Proposition: There exists a (non-canonical) isomorphism of schemes

$$
\pi: \underline{\operatorname{Aut}}_{\mathrm{uD}}\left(\underline{\bar{B}}(r) \oplus \underline{\bar{S}}^{m}\right) \cong \underline{\operatorname{Hom}}_{\mathrm{gD}}\left(\underline{\bar{S}}^{m}, \underline{\bar{B}}(r)\right) \times \underline{\operatorname{Aut}}_{\mathrm{uD}}\left(\underline{\bar{S}}^{m}\right) \times \underline{\operatorname{Aut}}_{\mathrm{uD}}(\underline{\bar{B}}(r)) .
$$

Proof : Consider an $R$-valued point $\varphi$ of $\underline{\operatorname{Hom}}_{\mathrm{gD}}\left(\underline{\bar{S}}^{m}, \underline{\bar{B}}(r)\right)$ for an arbitrary $k$ algebra $R$. The restriction of $\varphi$ to $\underline{\bar{S}}^{m} \otimes R$ factors by (3.11) through

$$
\varphi^{\prime}: \underline{\bar{S}}^{m} \otimes R \longrightarrow\left(\underline{\bar{B}}^{+}(r) \oplus \underline{\bar{S}}^{m}\right) \otimes R .
$$

As $\underline{B}^{+}(r)$ is the radical of $\underline{B}^{+}(r) \oplus \underline{S}^{m}$, one obtains two components

$$
\begin{aligned}
& \pi_{1}(\varphi) \in \underline{\operatorname{Hom}}_{\mathrm{gD}}\left(\underline{\bar{S}}^{m}, \underline{\bar{B}}^{+}(r)\right)(R)=\underline{\operatorname{Hom}}_{\mathrm{gD}}\left(\underline{\bar{S}}^{m}, \underline{\bar{B}}(r)\right)(R), \\
& \pi_{2}(\varphi) \in \underline{\operatorname{Aut}}_{\mathrm{uD}}\left(\underline{\bar{S}}^{m}\right)(R) .
\end{aligned}
$$

Observe that $\underline{\mathrm{Aut}}_{\mathrm{uD}}\left(\underline{\bar{S}}^{m}\right)$ is embedded in a canonical way in $\underline{\mathrm{Aut}}_{\mathrm{uD}}\left(\underline{\bar{B}}(r) \oplus \underline{\bar{S}}^{m}\right)$ and that it acts in a canonical way in $\underline{\operatorname{Hom}}_{\mathrm{gD}}\left(\underline{\bar{S}}^{m}, \underline{\bar{B}}(r)\right)$ from the right. The maps $\pi_{1}$ and $\pi_{2}$ are $\underline{\mathrm{Aut}}_{\mathrm{uD}}\left(\underline{\bar{S}}^{m}\right)$-equivariant morphism of schemes. This allows to construct a section $\sigma$ of the morphism

$$
\pi_{1} \times \pi_{2}: \underline{\mathrm{Aut}}_{\mathrm{uD}}\left(\underline{\bar{B}}(r) \oplus \underline{\bar{S}}^{m}\right) \longrightarrow \underline{\operatorname{Hom}}_{\mathrm{gD}}\left(\underline{\bar{S}}^{m}, \underline{\bar{B}}(r)\right) \times \underline{\mathrm{Aut}}_{\mathrm{uD}}\left(\underline{\bar{S}}^{m}\right) .
$$

For this consider an $R$-valued point $\left(\varphi_{1}, \varphi_{2}\right)$ of the right hand side. Let $S=\left(s_{i j}\right)$ (resp. $\left.T=\left(t_{i j}\right)\right)$ be the $(r \times m)$-matrix with entries in $R$ which describes $\varphi_{1} \circ \varphi_{2}^{-1}$ on the bases $g_{1}, \ldots, g_{m}$ and $e_{1}, \ldots, e_{r}$ (resp. on $h_{1}, \ldots, h_{m}$ and $f_{1}, \ldots, f_{r}$ ). Define an element $\psi$ in $\underline{\operatorname{Aut}}\left(\underline{\bar{B}}(r) \oplus \underline{\bar{S}}^{m}\right)(R)$ as follows: The 0-graded component is given by:

$$
\begin{aligned}
e_{j} & \mapsto e_{j}+(-1)^{r-1} \sum_{i} t_{j, i}\left(g_{i}+\frac{1}{2} \sum_{k} s_{k, i} e_{k}\right), \\
g_{j} & \mapsto g_{j}+\sum_{i} s_{i, j} e_{i},
\end{aligned}
$$

and the 1-graded component is given by:

$$
\begin{aligned}
f_{j} & \mapsto f_{j}+(-1)^{r} \sum_{i} s_{j, i}\left(h_{i}+\frac{1}{2} \sum_{k} t_{k, i} f_{k}\right), \\
h_{j} & \mapsto h_{j}+\sum_{i} t_{i, j} f_{i} .
\end{aligned}
$$

It is straight forward to see that $\psi$ is compatible with grading, form, and the operators $F$ and $V$. Further, we have $\pi_{1}(\psi)=\varphi_{1} \circ \varphi_{2}^{-1}$ and $\pi_{2}(\psi)=1$. If we set $\sigma\left(\varphi_{1}, \varphi_{2}\right)=$ $\psi \circ \varphi_{2}$, this defines the wanted section. 
For any $\varphi \in \underline{\operatorname{Aut}}_{\mathrm{uD}}\left(\underline{\bar{B}}(r) \oplus \underline{\bar{S}}^{m}\right)(R)$ we have

$$
\left.\varphi\right|_{\underline{\underline{S}}^{m}}=\left.\sigma\left(\pi_{1}(\varphi), \pi_{2}(\varphi)\right)\right|_{\underline{S}^{m}} .
$$

It follows that we have $\pi_{3}(\varphi):=\varphi^{-1} \sigma\left(\pi_{1}(\varphi), \pi_{2}(\varphi)\right) \in \underline{\text { Aut }}_{\mathrm{uD}}(\underline{\bar{B}}(r))(R)$ where we

consider $\underline{\operatorname{Aut}}_{\mathrm{uD}}(\underline{\bar{B}}(r))$ as a subgroup scheme of $\underline{\mathrm{Aut}}_{\mathrm{uD}}\left(\underline{\bar{B}}(r) \oplus \underline{\bar{S}}^{m}\right)$ in the canonical way. Then $\pi=\pi_{1} \times \pi_{2} \times \pi_{3}$ is the desired isomorphism.

(3.13) Corollary: For $1 \leq r \leq n$ we have

$$
\operatorname{dim} \underline{\operatorname{Aut}}\left(\underline{\bar{B}}(r) \oplus \underline{\bar{S}}^{n-r}\right)= \begin{cases}\frac{r}{2}-1, & \text { if } r \text { is even, } \\ n-\frac{r+1}{2}, & \text { if } r \text { is odd }\end{cases}
$$

\section{Structure of non-supersingular unitary Dieudonné modules}

(4.1) Lemma: Let $\underline{M}$ be a unitary Dieudonné module such that its reduction modulo $p$ can be decomposed as unitary Dieudonné spaces in $\underline{\bar{M}}^{\prime} \oplus \underline{\bar{S}}^{m}$ for some $m \geq 1$. Then there exists a decomposition of unitary Dieudonné modules $\underline{M}=\underline{M}^{\prime} \oplus \underline{S}^{m}$ inducing modulo $p$ the given decomposition.

Proof: We can assume that $m=1$. By $[\mathrm{Kr}]$ p. 56 we can find a decomposition of Dieudonné modules $M=M^{\prime \prime} \oplus S^{\prime}$ such that in $S^{\prime}$ there exists an $x$ with $F x=$ $V x \notin p S^{\prime}$. The image of $x$ modulo $p$ is a nonzero element in $(M / p M)_{0}$. Denote by $x_{0}$ the $M_{0}$-component of $x$. Then we still have $F x_{0}=V x_{0}$ and the Dieudonné module $S$ generated by $x_{0}$ is graded and superspecial. Further the restriction of $\langle$, to it is perfect as it is perfect modulo $p$. This way we get the superspecial unitary Dieudonné module $\underline{S}$. It lifts $\underline{\bar{S}}$. Its orthogonal complement $M^{\prime}$ is also a unitary Dieudonné-module $\underline{M}^{\prime}$, and it reduces modulo $p$ to $\underline{\bar{M}}^{\prime}$.

(4.2) Let $\underline{M}$ be a unitary Dieudonné module of signature $(n-1,1)$. Denote by $N$ its isocrystal. By (3.5) the reduction modulo $p$ of $\underline{M}$ is isomorphic to $\underline{\bar{B}}(r) \oplus \underline{\bar{S}}^{n-r}$ for some $r$ with $1 \leq r \leq n$.

Proposition: (1) $N$ is supersingular if and only if $r$ is odd.

(2) Assume that $N$ is not supersingular. Consider the decomposition $N=N^{\prime} \oplus N^{\prime \prime}$ with $N^{\prime}=\left(N_{\frac{1}{2}}\right)^{n-2 m}$ and $N^{\prime \prime}=N(m)$ (3.1). Set $M^{\prime}=M \cap N^{\prime}$ and $M^{\prime \prime}=$ 
$M \cap N^{\prime \prime}$. Then $M=M^{\prime} \oplus M^{\prime \prime}, M^{\prime}$ is superspecial, and the reduction of $M^{\prime \prime}$ with its induced Dieudonné space structure is isomorphic to $\underline{\bar{B}}(2 m)$.

Proof: For both assertions we can assume that $r=n$ by (4.1). The condition in (1) is sufficient by (3.3). Now let $r>0$ be even. We show that $N$ has no homogeneous supersingular subisocrystal. If such a supersingular isocrystal existed we would find a homogeneous element $x \in N$ of degree 0 such that $F x=V x$. By multiplying with a suitable power of $p$ we can assume that $x \in M_{0} \backslash p M_{0}$. Modulo $p$ we get a nonzero element $y \in \underline{\bar{B}}(r)$ such that $F y=V y$. This is a contradiction to $(3.10)(2)$.

By (1) we know that $r$ is even in the non-supersingular case and in its proof we showed that $N$ contains no supersingular subisocrystal. Therefore $M=M^{\prime \prime}$ (under the assumption $r=n$ ) and (2) follows.

(4.3) Let $\underline{M}$ be a unitary Dieudonné module of signature $(n-1,1)$ whose isocrystal is not supersingular. By (4.2) we have a decomposition of unitary Dieudonné modules $\underline{M}=\underline{M}^{\prime} \oplus \underline{M}^{\prime \prime}$ where $\underline{M}^{\prime}$ is superspecial and where $\underline{\bar{M}}^{\prime \prime} \cong \underline{\bar{B}}(2 m)$ (which is equivalent to the fact that its isocrystal is $N(m)$ ). The goal of the rest of this chapter is to give a precise description for those modules. In fact, we will show:

(4.4) Proposition: Let $\underline{M}$ be a unitary Dieudonné module whose reduction modulo $p$ is isomorphic to $\underline{\bar{B}}(2 m)$ for some $m \geq 1$. Then $\underline{M} \cong \underline{B}(2 m)$.

(4.5) More generally, for applications to $p$-isogenies we want to examine the set $\mathcal{M}$ of quasi-unitary (2.4) Dieudonné lattices $\left(M, F, V, M_{0} \oplus M_{1}\right)$ of $N(m)$ of signature $(2 m-1,1)$ such that

$$
M^{t}:=\{x \in N(m) \mid\langle x, M\rangle \subset W(k)\}=p^{l} M
$$

for some $l \in \mathbb{Z}$.

To do this we define the following generalized version of a braid. Let $m \geq 1, l$, and $0 \leq a \leq m-1$ integers. A generalized braid of lenght $2 m$, defect $l$, and jump $a$ is the following graded Dieudonné module $\left(M=M_{0} \oplus M_{1}, F, V\right)$ over $k$ together with a $W(k)$-bilinear alternating $W(k)_{\mathbb{Q}^{-v a l u e d}}$ form $\langle$,$\rangle :$

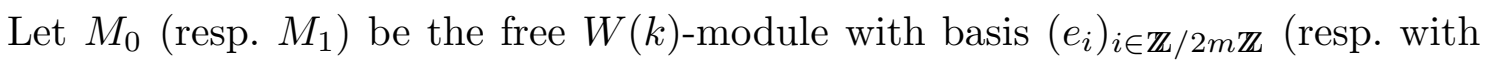

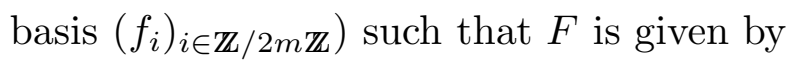

$$
\begin{aligned}
& F e_{i}=p^{\delta_{i, 1}} f_{i-1}, \\
& F f_{i}=p^{1-\delta_{i, 2 a+1}} e_{i-1} .
\end{aligned}
$$


Set $V=F^{-1} p$. Finally, define $\langle$,$\rangle by$

$$
\left\langle e_{i}, f_{j}\right\rangle= \begin{cases}(-1)^{i-1} p^{l+1} \delta_{i j}, & \text { if } i \leq 2 a, \\ (-1)^{i-1} p^{l} \delta_{i j}, & \text { if } i>2 a .\end{cases}
$$

Here and in the sequel we identify $\mathbb{Z} / 2 m \mathbb{Z}$ with $\{1, \ldots, 2 m\}$. A generalized braid is called integral if $l \geq 0$, i.e. $\langle M, M\rangle \subset W(k)$. It is called quasi-braid if $a=0$. We denote by $\mathcal{B}$ the set of generalized braids in $N(r)$.

(4.6) From now on let $\underline{N}=\left(N, F, V, N_{0} \oplus N_{1},\langle\rangle,\right)$ be a generalized braid of length $2 m$, defect $l$ and jump $a$. It follows from the definition that

$$
\lg _{W(k)}\left(N^{t} / N\right)=4(m l+a)
$$

In particular, a generalized braid is a braid iff $a=l=0$.

If $\underline{N}$ is a generalized braid, so is $p^{n} \underline{N}$ for all $n \in \mathbb{Z}$. In particular, every $\underline{M} \in \mathcal{M}$ contains some generalized braid.

(4.7) Lemma: Let $\underline{M}=\left(M, F, V, M_{0} \oplus M_{1},\langle\rangle,\right)$ be in $\mathcal{M}$ and let $\underline{N}$ be a generalized braid such that $N$ is properly contained in $M$. Then we have $V^{-1} N_{1} \subset M_{0}$ or $F^{-1} N_{1} \subset M_{0}$.

Proof : By replacing the alternating form of $M$ and $N$ by a suitable $p$-power we can assume that the form is perfect on $M$. As generalized braids have signature $(2 m-1,1)$, one has $\operatorname{dim} V^{-1} N_{1} / N_{0}=\operatorname{dim} F^{-1} N_{1} / N_{0}=1$. One can actually say more namely, if $e_{1}, \ldots, e_{2 m}, f_{1}, \ldots, f_{2 m}$ is a basis of $N$ as in (4.5), then

$$
V^{-1} N_{1}=N_{0}+W(k) p^{-1} e_{2 a},
$$

and

$$
F^{-1} N_{1}=N_{0}+W(k) p^{-1} e_{1}
$$

If $N$ is properly contained in $M$, then it is a non-perfect, though integral generalized braid, and we have $<p^{-1} e_{2 a}, N_{1}>\subset W(k)$ and $<p^{-1} e_{1}, N_{1}>\subset W(k)$. We conclude that

$$
V^{-1} N_{1} \subset N_{1}^{t}, \quad F^{-1} N_{1} \subset N_{1}^{t}
$$

where $N_{1}^{t}$ denotes the $W(k)$-lattice in $M_{0} \otimes \mathbb{Q}$ that is dual to $N_{1}$.

Now if $V^{-1} N_{1} \not \subset M_{0}$, then the first of the two inclusions

$$
M_{0} \subset V^{-1} N_{1}+M_{0} \subset V^{-1} M_{1},
$$


is proper, so the second one is not because $\operatorname{dim} V^{-1} M_{1} / M_{0}=1$. Consequently $V^{-1} M_{1}=V^{-1} N_{1}+M_{0} \subset N_{1}^{t}$ which gives the inclusion $N_{1} \subset F M_{0}$, by considering the dual lattices.

(4.8) We introduce two maps $\mathcal{F}, \mathcal{V}: \mathcal{B} \longrightarrow \mathcal{B}$ by setting

$$
\mathcal{F}(N)=N+F^{-1} N_{1}+V F^{-1} N_{1}, \quad \mathcal{V}(N)=N+V^{-1} N_{1}+F V^{-1} N_{1} .
$$

They satisfy $\mathcal{F} \mathcal{V}=\mathcal{V} \mathcal{F}, \mathcal{F}^{r} \mathcal{V}^{r}=p^{-1}$, and

$$
\lg _{W(k)}(\mathcal{F}(N) / N)=\lg _{W(k)}(\mathcal{V}(N) / N)=2
$$

(4.9) Proposition: Let $\underline{M}=\left(M, F, V, M_{0} \oplus M_{1},\langle\rangle,\right)$ be in $\mathcal{M}$ with $M^{t}=p^{\lambda} M$ and let $\underline{N}$ be a generalized braid of defect $l$ and jump a such that $N \subset M$. Then we have:

(1) There exist integers $\alpha, \beta \geq 0$ such that $M=\mathcal{F}^{\alpha} \mathcal{V}^{\beta} N$.

(2) We have $\alpha+\beta=r(l-\lambda)+a$.

(3) There exists only finitely many $\underline{M} \in \mathcal{M}$ with $N \subset M \subset N^{t}$.

(4) Every $\underline{M} \in \mathcal{M}$ is a quasi-braid, in particular (4.4) holds.

Proof: To prove (1) we make induction on $\lg _{W(k)}(M / N)$. If this length is positive, we have either $\mathcal{F}(N) \subset M$ or $\mathcal{V}(N) \subset M$ by (4.7) and we can apply the induction hypothesis to $\mathcal{F}(N) \subset M$ resp. $\mathcal{V}(N) \subset M$.

Clearly, (1) implies (4), and (2) follows from the equalities $[M: N]=\left[N^{t}: M^{t}\right]=$ $2(\alpha+\beta),\left[M^{t}: M\right]=4 r \lambda$, and $\left[N: N^{t}\right]=4(r l+a)$. Further, (3) follows from (1) and (2): The inclusions $N \subset M \subset N^{t}$ implies $\left|\left[M^{t}: M\right]\right| \leq\left[N^{t}: N\right]$, i.e. $|\lambda| \leq l$. With this restriction (and $\alpha, \beta \geq 0$ ) the equation in (2) has only finitely many solutions.

(4.10) Corollary: Let $\underline{M}$ be a unitary Dieudonnè module of signature $(n-1,1)$ such that the set of slopes of the associated isocrystal is $\{0,1 / 2,1\}$. Then

$$
\underline{M} \cong \underline{B}(2) \oplus \underline{S}^{n-2}
$$




\section{Newton polygon and Ekedahl-Oort stratification of the special fibre}

(5.1) From now on we make the assumption $(r, s)=(n-1,1)$ for the pair $(r, s)$ in (1.3). We denote by $\mathcal{M}$ the special fibre of $\mathcal{M}_{\mathcal{I}, K^{p}}$. Let $\mathbb{F}$ be an algebraic closure of $\kappa\left(O_{E_{v}}\right)$.

(5.2) Lemma: There exists an $\mathbb{F}$-valued point of $\mathcal{M}$ whose underlying abelian variety is supersingular.

Note that we do not really need this result in the sequel. In fact all arguments would simplify considerably if the supersingular locus of $\mathcal{M}$ were empty. Hence we remark for the proof only that we can work up to $\mathbb{Q}$-isogeny. Further it suffices to construct a supersingular abelian variety $A$ up to isogeny with $B$-action, then we can also find a polarization on $A$ by $[\mathrm{Ko}]$ 9.2. But this can be done using Honda-Tate theory (see loc. cit. §10).

(5.3) Theorem: Let $s, s^{\prime}: \operatorname{Spec}(k) \longrightarrow \mathcal{M}$ be two geometric points of characteristic $p$ corresponding to tuples $(A, \iota, \lambda, \bar{\eta})$ and $\left(A^{\prime}, \iota^{\prime}, \lambda^{\prime}, \bar{\eta}^{\prime}\right)$. Assume that $A$ is not supersingular. Then the following statements are equivalent:

(1) The $p$-divisible $\mathcal{I}$-modules $(A, \iota, \lambda)\left[p^{\infty}\right]$ and $\left(A^{\prime}, \lambda^{\prime}, \iota^{\prime}\right)\left[p^{\infty}\right]$ are isomorphic.

(2) The $B T_{1}$ 's with $O_{B}$-action and pairing $(A, \iota, \lambda)[p]$ and $\left(A^{\prime}, \lambda^{\prime}, \iota^{\prime}\right)[p]$ are isomorphic.

(3) The isocrystals with $B$-action and pairing associatd to $(A, \iota, \lambda)$ and $\left(A^{\prime}, \lambda^{\prime}, \iota^{\prime}\right)$ are isomorphic.

Proof: In each of the three cases (1) - (3) the non-supersingularity of $A$ implies that $A^{\prime}$ is not supersingular (this is obvious for (1) and (3) and it follows from (4.2)(1) if (2) holds). By (4.4) and (4.1), (1) and (2) are equivalent. Finally (4.2)(2) implies the equivalence of (2) and (3).

(5.4) Let $\operatorname{Spec}(k) \longrightarrow \mathcal{M}$ be a geometric point of characteristic $p$ corresponding to a tuple $(A, \iota, \lambda, \bar{\eta})$. The Dieudonné space of the $p$-torsion gives a unitary Dieudonné space of signature $(n-1,1)$ which is isomorphic to $\underline{\bar{B}}(\rho(\bar{s})) \oplus \underline{S}^{n-\rho(\bar{s})}$ for some integer $\rho(\bar{s}) \in\{1, \ldots, n\}$ by (3.5). This defines the Ekedahl-Oort stratification

$$
\mathcal{M}=\bigcup_{\rho=1}^{n} \mathcal{M}_{\rho}
$$


Now [We3] shows that all $\mathcal{M}_{\rho}$ are equi-dimensional and that if $\mathcal{M}_{\rho} \neq \emptyset$ we have

$$
\operatorname{codim}\left(\mathcal{M}_{\rho}, \mathcal{M}\right)=\operatorname{dim} \underline{\operatorname{Aut}}\left(\underline{\bar{B}}(\rho) \oplus \underline{\bar{S}}^{n-\rho}\right)= \begin{cases}\frac{\rho}{2}-1, & \text { if } \rho \text { is even, } \\ n-\frac{\rho+1}{2}, & \text { if } \rho \text { is odd }\end{cases}
$$

by (3.13). By (5.2) and (4.2) we know that the union of the $\mathcal{M}_{\rho}$ where $\rho$ runs through the odd numbers between 1 and $n$ is nonempty as this is precisely the supersingular locus.

In this case the Ekedahl-Oort stratification is finer than the Newton polygon stratification (4.2) which is given by

$$
\mathcal{M}=\mathcal{M}_{2} \cup \mathcal{M}_{4} \cup \ldots \cup \mathcal{M}_{2[n / 2]} \cup \bigcup_{\rho \text { odd }} \mathcal{M}_{\rho}
$$

Further de Jong and Oort have shown in [dJO] that going from one Newton polygon stratum $\mathcal{M}_{\rho}$ to the next smaller one, the codimension can only jump by 1 . It follows therefore from (5.4.1) that all Newton polygon strata are non-empty and equidimen-

sional. Further, $\mathcal{M}_{\rho^{\mathrm{ss}}}$ is dense in the supersingular locus $\bigcup_{\rho}$ odd $\mathcal{M}_{\rho}$ of $\mathcal{M}$ where $\rho^{\mathrm{ss}}$ is the maximal odd integer $\leq n$.

(5.5) Proposition: The dimension of the supersingular locus is equal to $[(n-1) / 2]$. Proof: This follows from (5.4).

(5.6) The stratum $\mathcal{M}_{2}$ of $\mathcal{M}$ is the $\mu$-ordinary stratum in the sense of [We1]. We also denote it by $\mathcal{M}^{\mu}$. If $\operatorname{Spec}(k) \longrightarrow \mathcal{M}^{\mu}$ is a geometric point corresponding to $(A, \iota, \lambda, \bar{\eta})$ with associated unitary Dieudonné module $\left(M, F, V, M=M_{0} \oplus M_{1},\langle\rangle,\right)$, the associated slope filtration of $(M, F, V)$ is actually a graduation

$$
M=M(0) \oplus M(1 / 2) \oplus M(1)
$$

and the $M(\lambda)$ are homogeneous Dieudonné submodules for $\lambda=0,1 / 2,1 ; M(0)$ and $M(1)$ are totally isotropic and orthogonal to $M(1 / 2)$ and they are free $O_{K} \otimes W(k)$ modules of rank $1 ; M(1 / 2)$ is orthogonal to $M(0) \oplus M(1)$ and it is isomorphic to $\underline{S}^{n-2}$.

\section{A generic model for the moduli space of $p$-isogenies}

(6.1) The goal in this chapter is to show that there are no components of $p$-Isog $\otimes$ $\kappa\left(E_{v}\right)(1.7)$ of dimension $<n-1$. For this we use Grothendieck-Messing theory to translate this problem into linear algebra. 
(6.2) Definition: Let $S$ be any $\mathbb{Z}_{p}$-scheme and let $c \geq 0$ be an integer. A $p$ isogeny pair over $S$ of type $\mathcal{I}$ of multiplicator $c$ or shorter an $\mathcal{I}$-pair over $S$ is a tuple $\left((M, \lambda),\left(M^{\prime}, \lambda^{\prime}\right), \alpha\right)$ where

- $M$ and $M^{\prime}$ are $O_{K} \otimes_{\mathbb{Z}_{p}} \mathcal{O}_{S^{-}}$-modules which are locally on $S$ free $\mathcal{O}_{S}$-modules of rank $2 n$.

- $\lambda: M \stackrel{\sim}{\longrightarrow} M^{\vee}$ and $\lambda^{\prime}: M^{\prime} \stackrel{\sim}{\longrightarrow} M^{\prime \vee}$ are isomorphisms of $O_{K} \otimes_{\mathbb{Z}_{p}} \mathcal{O}_{S}$-modules such that $\lambda^{\vee}=-\lambda$ and $\lambda^{\prime \vee}=-\lambda^{\prime}$. Here we denote by ()$^{\vee}$ the $\mathcal{O}_{S}$-linear dual which is endowed with an $O_{K}$-module structure via $b \cdot m^{\vee}(m)=m^{\vee}\left(b^{*} m\right)$ for $b \in O_{K}, m \in M$ and $m^{\vee} \in M^{\vee}$.

- $\alpha: M \longrightarrow M^{\prime}$ is an $O_{K} \otimes_{\mathbb{Z}_{p}} \mathcal{O}_{S}$-linear map such that

$$
\alpha^{\vee} \circ \lambda^{\prime} \circ \alpha=p^{c} \lambda \quad \text { and } \quad \alpha \circ \lambda^{-1} \circ \alpha^{\vee}=p^{c} \lambda^{\prime-1} .
$$

A morphism of $\mathcal{I}$-pairs is a commutative diagram

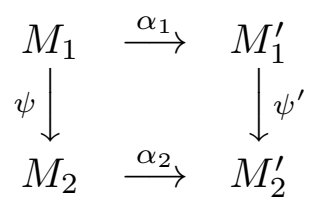

such that $\psi$ and $\psi^{\prime}$ are $O_{B} \otimes_{\mathbb{Z}_{(p)}} \mathcal{O}_{S^{-}}$-linear symplectic isomorphisms.

For varying $S$ the $\mathcal{I}$-pairs over $S$ with multiplicator $c$ define an Artin stack $\mathrm{dR}_{\mathcal{I}}^{c}$ over $\mathbb{Z}_{p}$, i.e. an algebraic stack in the sense of $[\mathrm{LM}]$.

(6.3) Let $S$ be a scheme. Denote by $\mathrm{P}^{c}(S)$ the category of tuples $\left(N, N^{\prime}, u, v\right)$ where $N$ and $N^{\prime}$ are locally free $\mathcal{O}_{S}$-modules of rank $n$ and where

$$
u: N \longrightarrow N^{\prime}, \quad v: N^{\prime} \longrightarrow N
$$

are $\mathcal{O}_{S}$-linear maps such that $v \circ u=p^{c} \operatorname{id}_{N}$ and $u \circ v=p^{c} \operatorname{id}_{N^{\prime}}$. A morphism

$$
\left(N_{1}, N_{1}^{\prime}, u_{1}, v_{1}\right) \longrightarrow\left(N_{2}, N_{2}^{\prime}, u_{2}, v_{2}\right)
$$

in this category is a pair of isomorphisms $\psi: N_{1} \longrightarrow N_{2}$ and $\psi^{\prime}: N_{1}^{\prime} \longrightarrow N_{2}^{\prime}$ such that the diagrams

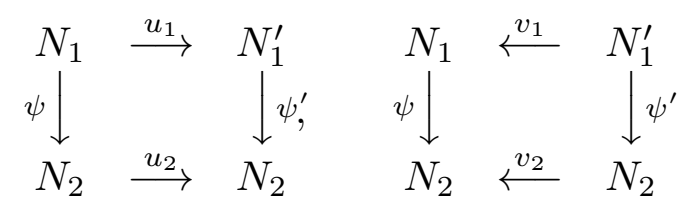

commute.

We have the obvious notion of a pullback with respect to a morphism $S^{\prime} \longrightarrow S$ and this makes $\mathrm{P}^{c}$ into an algebraic stack over $\mathbb{Z}$. 
(6.4) Let $S$ be an $O_{K}$-scheme and let $\left((M, \lambda),\left(M^{\prime}, \lambda^{\prime}\right), \alpha\right)$ be an $\mathcal{I}$-pair over $S$ of multiplicator $c$. The decomposition $O_{K} \otimes_{\mathbb{Z}_{p}} \mathcal{O}_{S}=\mathcal{O}_{S} \times \mathcal{O}_{S}$ induced by (2.1.2) gives a decomposition $M=M_{0} \oplus M_{1}$ such that $M_{i}$ is totally isotropic with repsect to the bilinear form given by $\lambda$. Hence $\lambda$ defines an isomorphisms $l_{0}: M_{0} \stackrel{\sim}{\longrightarrow} M_{1}^{\vee}$ and $l_{1}: M_{1} \stackrel{\sim}{\longrightarrow} M_{0}^{\vee}$. The same holds for $\left(M^{\prime}, \lambda^{\prime}\right)$. The map $\alpha$ is homogeneous with respect to the decomposition, i.e. $\alpha=\alpha_{0} \oplus \alpha_{1}$ for $\mathcal{O}_{S^{-}}$linear maps $\alpha_{i}: M_{i} \longrightarrow M_{i}^{\prime}$. The functor

$$
\mathcal{F}:\left((M, \lambda),\left(M^{\prime}, \lambda^{\prime}\right), \alpha\right) \mapsto\left(M_{0}, M_{0}^{\prime}, \alpha_{0}, l_{0}^{-1} \circ \alpha_{1}^{\vee} \circ l_{0}^{\prime}\right)
$$

defines an equivalence of the category of $\mathcal{I}$-pairs of multiplicator $c$ over $S$ with $\mathrm{P}_{S}^{c}$.

This equivalence is compatible with pullback via morphisms $S^{\prime} \longrightarrow S$. Hence we get an isomorphism of the algebraic stacks

$$
\mathcal{F}: \mathrm{dR}_{\mathcal{I}}^{c} \otimes_{\mathbb{Z}_{p}} O_{K} \stackrel{\sim}{\longrightarrow} \mathrm{P}^{c} \otimes_{\mathbb{Z}} O_{K}
$$

(6.5) We are going to study the special fibre of $p$-Isog ${ }^{c}$. More precisely we will study

$$
p \text {-Isog }{ }_{0}^{c}=p-\operatorname{Isog}{ }^{c} \otimes \kappa\left(O_{K}\right) .
$$

For $c=0$ each one of the morphisms $s, t: p$-Isog ${ }^{c} \longrightarrow \mathcal{M}_{\mathcal{I}, K^{p}}$ is an isomorphism. Hence we will assume form now on that $c>0$.

We will define a stratification of $p$-Isog ${ }_{0}^{c}$ by locally closed subschemes using the algebraic stack

$$
\mathrm{P}:=\mathrm{P}^{c} \otimes_{\mathbb{Z}} \kappa\left(O_{K}\right) .
$$

We remark that $\mathrm{P}$ is independent of $c>0$.

(6.6) Let $S$ be a $\kappa\left(O_{K}\right)$-scheme and let $f:(A, \iota, \lambda, \bar{\eta}) \longrightarrow\left(A^{\prime}, \iota^{\prime}, \lambda^{\prime}, \bar{\eta}^{\prime}\right)$ be an $S$ valued point of $p$-Isog ${ }^{c}$. By taking deRham cohomology we get via functoriality a homomorphism

$$
\alpha=H_{D R}(f): M=H_{D R}(A) \longrightarrow M^{\prime}=H_{D R}\left(A^{\prime}\right) .
$$

Further, $\lambda$ and $\iota$ endow $H_{D R}(A)$ with a homomorphisms $\lambda: M \longrightarrow M^{\vee}$ such that

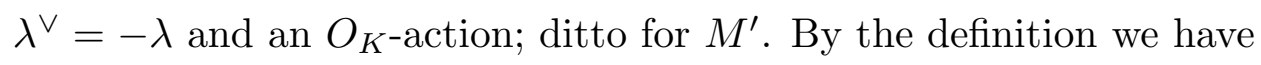

$$
\alpha^{\vee} \circ \lambda^{\prime} \circ \alpha=p^{c} \lambda=0 .
$$


By considering the dual $p$-isogeny $f^{\vee}$ and using the fact that $H_{D R}\left(f^{\vee}\right)=-\alpha^{\vee}$ we also have the relation

$$
\alpha \circ \lambda^{-1} \circ \alpha^{\vee}=p^{c} \lambda^{\prime-1}=0 .
$$

Altogether we get a morphism $p$-Isog ${ }_{0}^{c} \longrightarrow \mathrm{dR}_{\mathcal{I}}^{c} \otimes \kappa\left(O_{K}\right)$. By composition with the functor $\mathcal{F}(6.4)$ we obtain a morphism

$$
\Psi: p-\mathrm{I} \operatorname{sog}_{0}^{c} \longrightarrow \mathrm{P} .
$$

(6.7) Proposition: Let $S$ be a $\kappa\left(O_{K}\right)$-scheme and let $\tilde{N}_{1}=\left(N_{1}, N_{1}^{\prime}, u_{1}, v_{1}\right)$ and $\tilde{N}_{2}=\left(N_{2}, N_{2}^{\prime}, u_{2}, v_{2}\right)$ be two objects of $\mathrm{P}(S)$. Assume that $\operatorname{Im}\left(u_{i}\right)$ is locally a direct summand of $N^{\prime}$ of rank $m$ and that $\operatorname{Im}\left(v_{i}\right)$ is locally a direct summand of $N$ of rank $l$ (where $m$ and $l$ are nonnegative integers independant of $i=0,1) . \operatorname{Let} \underline{\operatorname{Isom}}_{S}\left(\tilde{N}_{1}, \tilde{N}_{2}\right)$ be the $S$-scheme of isomorphisms between $\tilde{N}_{1}$ and $\tilde{N}_{2}$.

(1) $\underline{\operatorname{Isom}}_{S}\left(\tilde{N}_{1}, \tilde{N}_{2}\right)$ admits Zariski locally on $S$ sections.

(2) $\underline{\operatorname{Isom}}_{S}\left(\tilde{N}_{1}, \tilde{N}_{2}\right)$ is smooth of relative dimension

$$
d(m, l):=n^{2}+(n-m-l)^{2}
$$

over $S$.

Proof: To prove (1) it suffices to show that there exists locally on $S$ a normal form of $\tilde{N}_{1}$ and $\tilde{N}_{2}$ which depends only on $m, l$. To shorten notations we write $\left(N, N^{\prime}, u, v\right)$ for $\tilde{N}_{i}$. Hence (1) follows from the following obvious lemma

Lemma: Let $S=\operatorname{Spec}(R)$ be a local scheme. There exist isomorphisms $\varphi: N \stackrel{\sim}{\longrightarrow}$ $R^{n}$ and $\varphi^{\prime}: N^{\prime} \stackrel{\sim}{\longrightarrow} R^{n}$ such that $u$ and $v$ are given by the matrices

$$
U=\left(\begin{array}{cc}
I_{m} & 0_{m, n-m} \\
0_{n-m, m} & 0_{n-m, n-m}
\end{array}\right), \quad V=\left(\begin{array}{ccc}
0_{m, m} & 0_{m, l} & 0_{m, n-m-l} \\
0_{l, m} & I_{l} & 0_{l, n-m-l} \\
0 & 0 & 0
\end{array}\right)
$$

where $0_{i, j}$ denotes the zero matrix of size $i \times j$.

Proof of the Proposition (continued): To prove (2) we can assume that $\tilde{N}_{1}=$ $\tilde{N}_{2}=: \tilde{N}$ is in the normal form of the lemma. It is clear that $\underline{\mathrm{Aut}}_{S}(\tilde{N})$ is smooth and a straight forward calculation gives its relative dimension.

(6.8) Lemma: Let $S$ be a scheme and let $\mathcal{E}$ be a quasicoherent $\mathcal{O}_{S}$-module of finite type. For every integer $n \geq 0$ denote by $\mathrm{rk}_{=n}(\mathcal{E})$ the functor on $(\mathrm{Sch} / S)$

$$
(f: T \longrightarrow S) \mapsto \begin{cases}\{f\}, & \text { if } f^{*} \mathcal{E} \text { is locally free of rank } n, \\ \emptyset, & \text { otherwise. }\end{cases}
$$


The functor $\mathrm{rk}_{=n}(\mathcal{E})$ is representable by a locally closed subscheme $Z_{n}$ of $S$ and the immersion $Z_{n} \longrightarrow S$ is of finite presentation if $\mathcal{E}$ is of finite presentation.

Proof: We can assume that $S=\operatorname{Spec}(A)$ is affine, $M$ the finitely generated $A$ module corresponding to $\mathcal{E}$. We define $Y_{n}$ as the closed subscheme given by the $(n-1)$-th Fitting ideal $\operatorname{Fitt}_{n-1}(M)$ of $M$ (see e.g. [Eis] §20.2) where we make the convention Fitt $_{-1}(M)=0$. If $M$ is of finite presentation the construction of $\operatorname{Fitt}_{n}(M)$ shows that the Fitting ideals are finitely generated. Set

$$
Z_{n}=Y_{n} \backslash Y_{n-1}
$$

This represents the functor $\mathrm{rk}_{=n}(\mathcal{F})$ by loc. cit. $20.5-8$.

(6.9) Let $\Xi=\Xi_{n}$ be the set of pairs $(m, l)$ of nonnegative integers such that $m+l \leq n$. For $\xi_{0}=\left(m_{0}, l_{0}\right)$ let $U_{\xi_{0}}$ be the set of pairs $(m, l) \in \Xi$ such that $m \geq m_{0}$ and $l \geq l_{0}$. We endow $\Xi$ with the topology generated by the $U_{\xi_{0}}$ for $\xi_{0} \in \Xi$. For $\xi=(m, l) \in \Xi$ we denote by $\mathrm{P}^{\xi}$ the substack of $\mathrm{P}$ of those $\left(N, N^{\prime}, u, v\right)$ where $\operatorname{Im}(u)$ is a direct summand of rank $m$ and $\operatorname{Im}(v)$ is a direct summand of rank $l$. Applying (6.8)(2) to the cokernels of $u$ and $v$ we see that the inclusion $\mathrm{P}^{\xi} \hookrightarrow \mathrm{P}$ is representable by an immersion. The underlying topological space of $\mathrm{P}^{\xi}$ consists of one point as all objects in $\mathrm{P}^{\xi}$ are locally isomorphic (6.7). By (6.8)(1) we further have (cf. [We3] 4.4):

Corollary: The underlying topological spaces of $\mathrm{P}$ is equal to $\Xi$.

(6.10) Let $S$ be a $\kappa\left(O_{K}\right)$-scheme. We denote by $\mathrm{M}(S)$ the category of tuples $\left(N, N^{\prime}, u, v, H, H^{\prime}\right)$ where $\left(N, N^{\prime}, u, v\right)$ is an object in $\mathrm{P}(S)$ and where $H \subset N$ and $H^{\prime} \subset N^{\prime}$ are locally direct summands of rank 1 . A morphism

$$
\left(N_{1}, N_{1}^{\prime}, u_{1}, v_{1}, H_{1}, H_{1}^{\prime}\right) \longrightarrow\left(N_{2}, N_{2}^{\prime}, u_{2}, v_{2}, H_{2}, H_{2}^{\prime}\right)
$$

in $\mathrm{M}(S)$ is a morphism $\left(\psi, \psi^{\prime}\right)$ in $\mathrm{P}(S)$ such that $\psi\left(H_{1}\right) \subset H_{2}$ and $\psi^{\prime}\left(H_{1}^{\prime}\right) \subset H_{2}^{\prime}$.

For varying $S$ we get the algebraic stack $\mathrm{M}$ over $\kappa\left(O_{K}\right)$.

(6.11) Associating to a $p$-isogeny the induced morphism on the deRham cohomology together with the Hodge filtration gives via the isomorphism $\mathcal{F}$ as in (6.6) a morphism

$$
\Phi: p-\operatorname{Isog}_{0}^{c} \longrightarrow \mathrm{M} .
$$

The forgetful functor $\left(N, N^{\prime}, u, v, H, H^{\prime}\right) \mapsto\left(N, N^{\prime}, u, v\right)$ defines a morphism

$$
\Pi: \mathrm{M} \longrightarrow \mathrm{P}
$$


and we have

$$
\Psi=\Pi \circ \Phi
$$

For $\xi \in \Xi$ we set

$$
\begin{aligned}
\mathrm{M}^{\xi} & :=\Pi^{-1}\left(\mathrm{P}^{\xi}\right), \\
\left(p-\operatorname{Isog}_{0}^{c}\right)^{\xi} & :=\Psi^{-1}\left(\mathrm{P}^{\xi}\right) .
\end{aligned}
$$

These is a locally closed substack of M resp. a locally closed subscheme of $p$-Isog ${ }_{0}^{c}$.

(6.12) For $\xi=(m, l) \in \Xi$ let $\tilde{N}_{0}^{\xi}=\left(N_{0}, N_{0}^{\prime}, u_{0}, v_{0}\right)$ be the object in $\mathrm{P}^{\xi}$ which has the normal form as in (6.7). Denote by $\widetilde{\mathrm{M}}^{\xi}$ the functor which associates to an $O_{K^{-}}$-scheme $S$ the set of isomorphism classes of tuples $\left(N, N^{\prime}, u, v, H, H^{\prime}, \alpha\right)$ where $\left(N, N^{\prime}, u, v, H, H^{\prime}\right)$ is an object in $\mathrm{M}^{\xi}$ and where $\alpha$ is an isomorphism $\left(N, N^{\prime}, u, v\right) \stackrel{\sim}{\longrightarrow}$ $\tilde{N}_{0}^{\xi} \otimes_{\kappa\left(O_{K}\right)} \mathcal{O}_{S}$ is isomorphic to the scheme whose $S$-valued points are given by pairs $\left(H, H^{\prime}\right)$ where $H \subset N_{0} \otimes \mathcal{O}_{S}$ and $H^{\prime} \subset N_{0}^{\prime} \otimes \mathcal{O}_{S}$ are locally direct summands of rank 1 such that $\left(u_{0} \otimes \mathrm{id}\right)(H) \subset H^{\prime}$ and $\left(v_{0} \otimes \mathrm{id}\right)\left(H^{\prime}\right) \subset H$. Hence it is representable by a projective scheme over $\kappa\left(O_{K}\right)$. By $(6.7)$ the canonical morphism

$$
\widetilde{\mathrm{M}}^{\xi} \longrightarrow \mathrm{M}^{\xi}
$$

is a torseur under the smooth $\kappa\left(O_{K}\right)$-group scheme $\underline{\operatorname{Aut}}\left(\tilde{N}_{0}^{\xi}\right)$.

We consider $\widetilde{\mathrm{M}}^{\xi}$ as a closed subscheme of $\mathbb{P}\left(N_{0}\right) \times \mathbb{P}\left(N_{0}^{\prime}\right)$. It has three distinguished closed subschemes $Z, Z^{\prime}$ and $Z^{\prime \prime}: Z$ is the closure of the locus where $\left(u_{0} \otimes \mathrm{id}\right)(H)$ is locally a direct summand of rank 1 , and it is isomorphic to $\mathbb{P}\left(N_{0}\right)$ via the first projection $\widetilde{\mathrm{M}}^{\xi} \longrightarrow \mathbb{P}\left(N_{0}\right) ; Z^{\prime}$ is the closure of the locus where $\left(v_{0} \otimes \mathrm{id}\right)\left(H^{\prime}\right)$ is locally a direct summand of rank 1 , and it is isomorphic to $\mathbb{P}\left(N_{0}^{\prime}\right)$ via the second projection $Z \longrightarrow \mathbb{P}\left(N_{0}^{\prime}\right) ; Z^{\prime \prime}$ is the locus where $\left(u_{0} \otimes \mathrm{id}\right)(H)=(0)$ and $\left(v_{0} \otimes \mathrm{id}\right)\left(H^{\prime}\right)=(0)$ and it is isomorphic to $\mathbb{P}\left(\operatorname{Ker}\left(u_{0}\right)\right) \times \mathbb{P}\left(\operatorname{Ker}\left(v_{0}\right)\right)$ via the embedding $Z \hookrightarrow \mathbb{P}\left(N_{0}\right) \times \mathbb{P}\left(N_{0}^{\prime}\right)$. Further we have $Z \cap Z^{\prime \prime}=\mathbb{P}\left(\operatorname{Ker}\left(u_{0}\right)\right) \times \mathbb{P}\left(\operatorname{Im}\left(u_{0}\right)\right) \subset Z^{\prime \prime}$ and $Z^{\prime} \cap Z^{\prime \prime}=\mathbb{P}\left(\operatorname{Im}\left(v_{0}\right)\right) \times$ $\mathbb{P}\left(\operatorname{Ker}\left(v_{0}\right)\right) \subset Z^{\prime \prime}$. Hence we get:

Lemma: If $m=n$ or $l=n$, we have $\widetilde{\mathrm{M}}^{\xi} \cong \mathbb{P}^{n}$. If $m+l=n$ and $m, l \geq 1, \widetilde{\mathrm{M}}^{\xi}$ has the two components $Z$ and $Z^{\prime}$ and their intersection has codimension 1 in $Z$ and $Z^{\prime}$. If $m+l<n$ we have three components, namely $Z, Z^{\prime}$ and $Z^{\prime \prime}$. In any case we have $\operatorname{dim}\left(\tilde{M}^{\xi}\right) \geq n-1$.

(6.13) Proposition: Fix a $\xi=(m, l)$ in $\Xi$. Let $U$ be an open subscheme of $p$-Isog ${ }_{0}^{c}$ which is contained in $\left(p-\operatorname{Isog}_{0}^{c}\right)^{\xi}$. The morphism

$$
\Phi_{U}: U \longrightarrow \mathrm{M}^{\xi}
$$


induced by $\Phi$ is smooth of relative dimension $d(m, l)(6.7)$.

Proof: We first prove smoothness using Grothendieck-Messing theory. As $\Phi_{U}$ is a morphism of finite type between noetherian Artin stacks it suffices to show that for all local Artinian rings $R$ and for all quotients $R_{0}$ of $R$ defined by an ideal $I$ of square zero and for all commutative diagrams

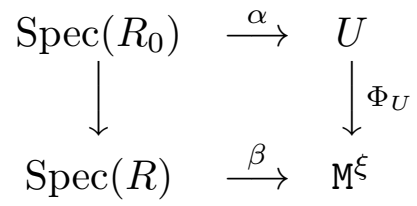

there exists a morphism $\operatorname{Spec}(R) \longrightarrow U$ which commutes with the diagram. As $U$ is open in $p$-Isog ${ }_{0}^{c}$ it suffices to construct a morphism $\operatorname{Spec}(A) \longrightarrow p$-Isog $\operatorname{sog}_{0}^{c}$ which makes the corresponding diagram commutative. As $I^{2}=(0)$ we can equip $I$ with a PD-structure. The morphism $\alpha: \operatorname{Spec}\left(A_{0}\right) \longrightarrow U$ corresponds to a $p$-isogeny $f:\left(A_{1}, \iota_{1}, \lambda_{1}, \bar{\eta}_{1}\right) \longrightarrow\left(A_{2}, \iota_{2}, \lambda_{2}, \bar{\eta}_{2}\right)$ over $R_{0}$, taking the corresponding morphisms of crystals and evaluating it in $R$ we get an $R$-valued point of $\mathrm{P}^{\xi}$. By (6.7) we find an isomorphism of this $R$-valued point of $\mathrm{P}^{\xi}$ with the $R$-valued point given by the composition of $\beta$ with $\mathrm{M}^{\xi} \longrightarrow \mathrm{P}^{\xi}$ induced by $\Pi$. Via this isomorphism the morphism $\beta$ defines a lifting of the Hodge filtration in the crystal of $f$ hence by Grothendieck-Messing theory the desired morphism $\operatorname{Spec}(R) \longrightarrow p$-Isog.

To prove the assertion about the relative dimension let $\tilde{U}$ be the fibre product of $U$ and $\widetilde{\mathrm{M}}^{\xi}$ over $\mathrm{M}^{\xi}$. Then $\tilde{U} \longrightarrow U$ is an $\underline{\operatorname{Aut}}\left(\tilde{N}_{0}^{\xi}\right)$-torseur and hence smooth of relative dimension $d(\xi)(6.7)$. In order to show that the smooth morphism $\tilde{U} \longrightarrow \widetilde{\mathrm{M}}^{\xi}$ has relative dimension $d(\xi)$, it suffices to prove that if $\tilde{u}$ is some $k$-valued point of $\tilde{U}$ with image $u$ resp. $\tilde{m}$ in $U$ resp. $\widetilde{\mathrm{M}}^{\xi}$ we have $\operatorname{dim}_{k} T_{u}(U)=\operatorname{dim}_{k} T_{\tilde{m}} \widetilde{\mathrm{M}}^{\xi}$ which again follows as above from (6.7) and Grothendieck-Messing theory applied to $R_{0}=k$ and $R=k[\varepsilon] / \varepsilon^{2}$.

(6.14) Corollary: We keep the notations of the proof of (6.13). For all closed points $u \in U$ there exists an étale neighborhood $U^{\prime}$ of $u$ and a section $U^{\prime} \longrightarrow \tilde{U}$ such that the composition $U^{\prime} \longrightarrow \tilde{U} \longrightarrow \widetilde{\mathrm{M}}^{\xi}$ is étale. In particular, for all closed points $u \in U$ there exists an $\tilde{m} \in \widetilde{\mathbb{M}}^{\xi}$ such that $\operatorname{dim}_{u}(U)=\operatorname{dim}_{\tilde{m}} \widetilde{\mathrm{M}}^{\xi}$.

Proof: As we need only the second assertion, we omit the proof of the more precise statement. To prove the second assertion we use the notations of the proof of (6.13). We have seen that there are surjective smooth morphism $\tilde{U} \longrightarrow U$ and $\tilde{U} \longrightarrow \widetilde{\mathrm{M}}^{\xi}$ of the same relative dimension $d(\xi)$ which implies the claim. 
(6.15) Proposition: Every component of $p-\mathrm{Isog}^{c} \otimes \kappa\left(O_{E, \nu}\right)$ has dimension $\geq n-1$.

Proof: We can assume that $c>0$. It suffices to show that every component of $p$-Isog ${ }_{0}^{c}$ has dimension $\geq n-1$. Let $X$ be a component of $p$-Isog $\operatorname{sog}_{0}^{c}$ and let $\xi$ be the element of $\Xi$ such that the generic point $\eta$ of $X$ lies in $\left(p-I \operatorname{sog}_{0}^{c}\right)^{\xi}$. Hence $\left(p-\operatorname{Isog}{ }_{0}^{c}\right)^{\xi} \cap X$ is open in $X$. If $U$ is an open subset of $\left(p-\operatorname{Isog}_{0}^{c}\right)^{\xi} \cap X$ which does not meet any other component of $p$-I $\operatorname{sog}_{0}^{c}, U$ is open in $p$-Isog ${ }_{0}^{c}$ and dense in $X$. We can apply (6.14) to $U$ and the claim follows from the fact that every component of $\widetilde{\mathrm{M}}^{\xi}$ has dimension $\geq n-1$ (6.12).

\section{Source and target morphism}

(7.1) To shorten notations we set $Y=Y^{c}=p$-Isog ${ }^{c} \otimes \kappa\left(O_{E_{v}}\right)$. Let $Y^{\mu} \subset Y$ be the $\mu$-ordinary locus and denote by $Y^{\text {nss }}$ (resp. $\mathcal{M}^{\text {nss }}$ ) the non-supersingular locus of $Y$ (resp. of the special fibre $\mathcal{M}$ of $\left.\mathcal{M}_{\mathcal{I}, K^{p}}\right)$. Let $k$ be an algebraic closure of $\kappa\left(O_{K}\right)$ and let $\bar{x}=(A, \iota, \lambda, \bar{\eta})$ be a $k$-valued point of $\mathcal{M}^{\text {nss }}$. Denote by $Y_{\bar{x}}^{\text {nss }}$ the fibre of the source morphism $Y^{\mathrm{nss}} \longrightarrow \mathcal{M}^{\mathrm{nss}}$ in $\bar{x}$.

(7.2) Proposition: The number of $k$-valued points in $Y_{\bar{x}}^{\text {nss }}$ is finite and depends only on the isogeny class of $\bar{x}$.

Proof: Let $\underline{M}=\left(M, F, V, M=M_{0} \oplus M_{1},\langle\rangle,\right)$ be a unitary Dieudonné module associated to $(A, \iota, \lambda, \bar{\eta})$. To give a $k$-valued point of $Y_{\bar{x}}$ is the same as to give an equivalence class of homomorphisms

$$
\alpha: \underline{M} \longrightarrow \underline{M^{\prime}}
$$

of graded Dieudonné modules such that $\langle\alpha(x), \alpha(y)\rangle^{\prime}=p^{c}\langle x, y\rangle$ where $\underline{M}^{\prime}$ is another unitary Dieudonné module. Here we call $\left(\alpha_{1}, \underline{M}_{1}^{\prime}\right)$ and $\left(\alpha_{2}, \underline{M}_{2}^{\prime}\right)$ equivalent if there exists an isomorphism $\psi: \underline{M}_{1}^{\prime} \stackrel{\sim}{\longrightarrow} \underline{M}_{2}^{\prime}$ such that $\psi \circ \alpha_{1}=\alpha_{2}$. We have decompositions $\underline{M}=B \oplus S$ and $\underline{M^{\prime}}=B^{\prime} \oplus S^{\prime}$ of unitary Dieudonné modules where $B \cong B^{\prime} \cong \underline{B}(r)$ and $S \cong S^{\prime} \cong \underline{S}^{n-r}$ for some even integer $2 \leq r \leq n$ (4.1) and (4.4). As the isogeny type depends only on $r$ (3.1), the second claim follows.

The homomorphism $\alpha$ respects this decomposition. Hence to show that there are only finitely many $\alpha$ up to equivalence as in $(*)$ we can assume that either $\underline{M} \cong \underline{M}^{\prime} \cong$ $\underline{B}(r)$ or that $\underline{M} \cong \underline{M}^{\prime} \cong \underline{S}^{n-r}$. The claim for the first case follows from (4.9). Hence we can assume that we are in the second case. It suffices to show that for any integer $m \geq 1$ there are only finitely many equivalence classes of homomorphisms of graded 
Dieudonné modules $\beta$ : $\underline{S}^{m} \longrightarrow \underline{S}^{m}$ such that the $W(k)$-length of the cokernel of $\beta$ is equal to $\mathrm{cm} / 2$. But any homomorphism $\beta$ is determined by $f:=\left.\beta\right|_{S_{0}^{m}}: S_{0}^{m} \longrightarrow S_{0}^{m}$. As $\beta$ commutes with $F$ and $V$ we have $f^{\sigma^{2}}=f$, i.e. $f \in M_{m}\left(W\left(\mathbb{F}_{p^{2}}\right)\right)$. The compatibility with the alternating forms translates into the condition ${ }^{t} f^{\sigma} f=p^{c}$. Two such elements $f_{1}$ and $f_{2}$ are equivalent if and only if there exists an $h \in J\left(\mathbb{Z}_{p}\right)$ where $J$ is the $\mathbb{Z}_{p}$-group scheme

$$
J=\left\{g \in \operatorname{Res}_{W\left(\mathbb{F}_{p^{2}}\right) / \mathbb{Z}_{p}} G L_{m} \mid{ }^{t} g^{\sigma} g=1\right\}
$$

such that $h f_{1}=f_{2}$. Hence we have to show that the $J$-orbits on

$$
X_{c}=\left\{\left.f \in M_{m}\left(W\left(\mathbb{F}_{p^{2}}\right)\right)\right|^{t} f^{\sigma} f=p^{c}\right\}
$$

are finite. Every $J\left(\mathbb{Z}_{p}\right)$-orbit on $X_{c}$ is open as $J\left(\mathbb{Z}_{p}\right)$ is open in $J\left(\mathbb{Q}_{p}\right)$ and for any two points $x, x^{\prime} \in X_{c}$ there exists a $g \in J\left(\mathbb{Q}_{p}\right)$ such that $g x=x^{\prime}$. As $X_{c}$ is compact, $X_{c} / J\left(\mathbb{Z}_{p}\right)$ is compact and discrete and therefore finite.

(7.3) Corollary: The restriction of source and target morphism to $Y^{\text {nss }}$ is a finite morphism $Y^{\mathrm{nss}} \longrightarrow \mathcal{M}^{\mathrm{nss}}$.

Proof: The source morphism is quasi-finite by (7.2), hence it is finite because it is also proper. By duality the same holds for the target morphism.

(7.4) Let $f:(A, \iota, \lambda, \bar{\eta}) \longrightarrow\left(A^{\prime}, \iota^{\prime}, \lambda^{\prime}, \bar{\eta}^{\prime}\right)$ be a $k$-valued point of $p$-Isog ${ }^{\mu}$ where $k$ is an algebraically closed field. Let $\mathcal{C}$ be the category of pairs $(R, \alpha)$ where $R$ is a local Artinian $O_{E_{v}}$-algebra and where $\alpha$ is an isomorphism $\kappa(R) \stackrel{\sim}{\longrightarrow} k$. We denote by $\operatorname{Def}(f)$ the functor of deformations of $f$ to $\mathcal{C}$. To give a deformation of $f$ is the same as to give a deformation of the morphism

$$
f: \underline{X}=(X, \iota, \lambda) \longrightarrow \underline{X}^{\prime}=\left(X^{\prime}, \iota^{\prime}, \lambda^{\prime}\right)
$$

of $p$-divisible $\mathcal{I}$-modules associated to $f(2.2)$ by the theorem of Serre-Tate.

There exists a unique decomposition $X=X_{\text {inf }} \times X_{\text {ét }}$ where $X_{\text {inf }}$ and $X_{\text {ét }}$ are the infinitesimal resp. the étale part of $X$ with induced $O_{K}$-action $\iota_{\text {inf }}$ and $\iota_{\text {ét }}$. Further $\lambda$ induces isomorphisms $\lambda_{\text {inf }}: X_{\text {inf }} \stackrel{\sim}{\longrightarrow}\left(X^{\vee}\right)_{\text {inf }}$ and $\lambda_{\text {ét }}: X_{\text {ét }} \stackrel{\sim}{\longrightarrow}\left(X^{\vee}\right)_{\text {ét. }}$ We set $\underline{X}_{\text {inf }}=\left(X_{\text {inf }}, \iota_{\text {inf }}, \lambda_{\text {inf }}\right), \underline{X}_{\text {ét }}=\left(X_{\text {ét }}, \iota_{\text {ét }}, \lambda_{\text {ét }}\right)$ and $\underline{X}_{\mathrm{bi}}=\left(\left(\underline{X}_{\text {inf }} \vee\right)_{\text {inf }}\right)^{\vee}$. We make the same definitions of $\underline{X}^{\prime}$ and get induced morphisms $f_{\text {inf }}, f_{\text {ét }}$ and $f_{\mathrm{bi}}$.

We have canonical morphisms $\operatorname{Def}(f) \longrightarrow \operatorname{Def}\left(f_{\text {inf }}\right) \longrightarrow \operatorname{Def}\left(f_{\text {bi }}\right)$. Further, sending a deformation to its source (resp. target) gives morphisms $\operatorname{Def}(f) \longrightarrow \operatorname{Def}(\underline{X})$, 
$\operatorname{Def}\left(f_{\text {inf }}\right) \longrightarrow \operatorname{Def}\left(\underline{X}_{\text {inf }}\right)$ and $\operatorname{Def}\left(f_{\mathrm{bi}}\right) \longrightarrow \operatorname{Def}\left(\underline{X}_{\mathrm{bi}}\right)\left(\right.$ resp. $\operatorname{Def}(f) \longrightarrow \operatorname{Def}\left(\underline{X}^{\prime}\right)$, $\operatorname{Def}\left(f_{\mathrm{inf}}\right) \longrightarrow \operatorname{Def}\left(\underline{X}_{\mathrm{inf}}^{\prime}\right)$ and $\left.\operatorname{Def}\left(f_{\mathrm{bi}}\right) \longrightarrow \operatorname{Def}\left(\underline{X}_{\mathrm{bi}}^{\prime}\right)\right)$.

(7.5) Proposition: With the notations of (7.4) there are the following relations and descriptions of these functors:

(1) The induced morphisms

$$
\begin{aligned}
\operatorname{Def}(f) & \longrightarrow \operatorname{Def}(\underline{X}) \times \operatorname{Def}\left(\underline{X}^{\prime}\right), \\
\operatorname{Def}\left(f_{\mathrm{inf}}\right) & \longrightarrow \operatorname{Def}\left(\underline{X}_{\mathrm{inf}}\right) \times \operatorname{Def}\left(\underline{X}_{\mathrm{inf}}^{\prime}\right), \\
\operatorname{Def}\left(f_{\mathrm{bi}}\right) & \longrightarrow \operatorname{Def}\left(\underline{X}_{\mathrm{bi}}\right) \times \operatorname{Def}\left(\underline{X}_{\mathrm{bi}}^{\prime}\right)
\end{aligned}
$$

are representable by closed immersions.

(2) For $(R, \alpha)$ in $\mathcal{C}$ the inverse image of every element in $\operatorname{Def}\left(\underline{X}_{\text {inf }}\right)(R, \alpha)$ under the canonical map $\operatorname{Def}(\underline{X}) \longrightarrow \operatorname{Def}\left(\underline{X}_{\text {inf }}\right)$ is nonempty and a principal homogenous space under

$$
T_{\mathrm{inf}}(X)(R):=\operatorname{Hom}_{\mathbb{Z}_{p}}^{\mathrm{Sym}}\left(T_{p}(X) \otimes_{O_{K}} T_{p}(X), \hat{\mathbb{G}}_{m}(R)\right) .
$$

(3) The inverse image of every element $\underline{\tilde{X}}_{\mathrm{bi}}$ in $\operatorname{Def}\left(\underline{X}_{\mathrm{bi}}\right)(R, \alpha)$ under the canonical map $\operatorname{Def}\left(\underline{X}_{\mathrm{inf}}\right) \longrightarrow \operatorname{Def}\left(\underline{X}_{\mathrm{bi}}\right)$ is nonempty and isomorphic to

$$
T_{\mathrm{bi}}\left(X, \tilde{X}_{\mathrm{bi}}\right)(R):=\operatorname{Hom}_{O_{K}}\left(T_{p}(X), \underline{\tilde{X}}_{\mathrm{bi}}(R)\right) .
$$

(4) $\operatorname{Def}\left(\underline{X}_{\mathrm{bi}}\right)$ is trivial, i.e. equal to $\operatorname{Spf}(W(k))$.

(5) Set ${ }^{t} f=\lambda^{-1} \circ f^{\vee} \circ \lambda^{\prime}: X^{\prime} \longrightarrow X$. The inverse image of an element in $\operatorname{Def}\left(f_{\text {inf }}\right)(R, \alpha)$ under the canonical map $\operatorname{Def}(f) \longrightarrow \operatorname{Def}\left(f_{\text {inf }}\right)$ is non-empty and naturally a torsor under

$$
\left\{\left(\beta, \beta^{\prime}\right) \in T_{\mathrm{inf}}(X)(R) \times T_{\mathrm{inf}}\left(X^{\prime}\right)(R) \mid \beta\left(T_{p}\left({ }^{t} f\right)\left(x^{\prime}\right), x\right)=\beta^{\prime}\left(x^{\prime}, T_{p}(f)(x)\right)\right\} .
$$

(6) The inverse image of $\tilde{f}_{\mathrm{bi}} \in \operatorname{Def}\left(f_{\mathrm{bi}}\right)(R, \alpha)$ under the map $\operatorname{Def}\left(f_{\mathrm{inf}}\right) \longrightarrow$ $\operatorname{Def}\left(f_{\mathrm{bi}}\right)$ is canonically isomorphic to

$$
\left\{\left(\gamma, \gamma^{\prime}\right) \in T_{\mathrm{bi}}\left(X, \tilde{X}_{\mathrm{bi}}\right)(R) \times T_{\mathrm{bi}}\left(X, \tilde{X}_{\mathrm{bi}}\right)(R) \mid \tilde{f}_{\mathrm{bi}}(R) \circ \gamma=\gamma^{\prime} \circ T_{p}(f)\right\} .
$$

(7) $\operatorname{Def}\left(f_{\text {bi }}\right)$ is trivial, i.e. equal to $\operatorname{Spf}(W(k))$. 
Proof: The assertions (1), (2), (3), (5) and (6) are standard (cf. [CN]) and hold in much greater generality. We omit the proofs.

As $\operatorname{Def}\left(\underline{X}_{\mathrm{bi}}\right)$ is pro-representable and formally smooth, in order to prove (4), it suffices to show that its tangent space $t_{\operatorname{Def}\left(\underline{X}_{\mathrm{bi}}\right)}$ is zero-dimensional. This tangent space is canonically isomorphic to

$$
\left\{u \in \operatorname{Hom}_{O_{K} \otimes_{\mathbb{Z}_{p}} k}\left(t_{X^{\vee}}^{*}, t_{X}\right) \mid\left(t_{\lambda}^{-1}\right) \circ\left(-u^{*}\right) \circ\left(t_{\lambda}^{*}\right)=u\right\}
$$

where $t_{X \vee}^{*}$ denotes the $k$-dual of $t_{X \vee}$ which is endowed with an $O_{K}$-action via $\left(a \cdot t^{*}\right)(t)=t^{*}(\sigma(a) t)$ for $a \in O_{K}, t^{*} \in t_{X^{\vee}}^{*}$ and $t \in t_{X^{\vee}}$. The $O_{K} \otimes k$-action defines decompositions $t_{X}=t_{0} \oplus t_{1}$ and $t_{X \vee}=t_{0}^{\prime} \oplus t_{1}^{\prime}$. As the Dieudonné modules of $\underline{X}_{\mathrm{bi}}$ and $\underline{X}_{\mathrm{bi}}{ }^{\vee}$ are isomorphic to $\underline{S}^{n-2}$ we see that $t_{1}=t_{1}^{\prime}=0$. By the definition of the $O_{K^{-}}$-action on $t_{X^{\vee}}^{*}$ an $O_{K} \otimes k$-linear map $t_{X^{\vee}}^{*} \longrightarrow t_{X}$ corresponds to a pair of maps $\left(t_{1}^{\prime}\right)^{*} \longrightarrow t_{0}$ and $\left(t_{0}^{\prime}\right)^{*} \longrightarrow t_{1}$ which shows

$$
\operatorname{Hom}_{O_{K} \otimes \mathbb{Z}_{p} k}\left(t_{X \vee}^{*}, t_{X}\right)=0 .
$$

Let us prove (7). As $\underline{X}_{\mathrm{bi}}$ and $\underline{X}_{\mathrm{bi}}^{\prime}$ are isomorphic we can assume that $\underline{X}_{\mathrm{bi}}=\underline{X}_{\mathrm{bi}}^{\prime}$. For every $(R, \alpha)$ in $\mathcal{C}$ denote by $\left(\underline{X}_{\mathrm{bi}}\right)_{R}$ the unique deformation of $\underline{X}_{\mathrm{bi}}$ to $R$. By (1) we know that there exists at most one deformation of $f_{\mathrm{bi}}$ to a morphism $\left(\underline{X}_{\mathrm{bi}}\right)_{R} \longrightarrow$ $\left(\underline{X}_{\mathrm{bi}}\right)_{R}$. Hence we have to show the existence of such a morphism. The $p$-divisible group $\underline{X}_{\mathrm{bi}}$ is isomorphic to the $(n-2)$-fold product of $p$-divisible $\mathcal{I}$-modules whose Dieudonné modules are isomorpic to $\underline{S}$. Therefore we can assume $n=3$. We will now use Zink's display theory [Zi2]. The display of $\left(\underline{X}_{\mathrm{bi}}\right)_{R}$ is given by $\left(P, Q, F, V^{-1}\right)$ where $P$ is a the free $W(R)$-module with base $(e, f)$, where $Q$ is the submodule $\tau(1) W(R) e \oplus W(R) f$ and where $F$ and $V^{-1}$ are given by

$$
F e=-f, \quad F f=\tau(1) e, \quad V^{-1} \tau(1) e=-f, \quad V^{-1} f=e .
$$

The $O_{K}$-action is given by the decomposition $P=P_{0} \oplus P_{1}$ with $P_{0}=W(R) e$ and $P_{1}=W(R) f$, and the polarization is defined by a perfect alternating form $\langle$,$\rangle such$ that $\langle e, f\rangle=1$. Indeed, this is clear for $R=k$. For arbitrary $R$ this is a deformation of the display over $k$. As this deformation is unique, the given display with $O_{K}$-action and polarization is the display of $\left(\underline{X}_{\mathrm{bi}}\right)_{R}$. To give a $p$-isogeny $\left(\underline{X}_{\mathrm{bi}}\right)_{R} \longrightarrow\left(\underline{X}_{\mathrm{bi}}\right)_{R}$ of multiplicator $c$ is the same as to give a homogeneous morphism $\varphi:\left(P, Q, F, V^{-1}\right) \longrightarrow$ $\left(P, Q, F, V^{-1}\right)$ such that $\langle\varphi(x), \varphi(y)\rangle=p^{c}\langle x, y\rangle$. Every such morphism is determined by $\varphi(e)$ which has to be of the form $w e$ for some $w \in W(R)$ because of the homogenity of $\varphi$. The condition that $\varphi$ is compatible with $F$ and $V^{-1}$ is equivalent to the condition $w=\sigma^{2}(w)$ and the compatability with the form up to $p^{c}$ means $w \sigma(w)=p^{c}$. Hence it 
suffices to show that for any $w_{0} \in W(k)$ with these properties there exists a $w \in W(R)$ with these properties that lifts $w_{0}$. As for every local Artinian ring $R$ with residue field $k$ there is a unique homomorphism $W(k) \longrightarrow R$ we can assume that $R=W(k)$. But then $\Delta\left(w_{0}\right)$ does the job, where $\Delta: W(k) \longrightarrow W(W(k))$ is the homomorphism of Cartier-Dieudonné, i.e. the unique homomorphism of rings which is compatible with Frobenius (see e.g. [Laz] VII, §4).

(7.6) Let $k$ be an algebraically closed field of characteristic $p$ and let $R$ be a complete local noetherian $E$-algebra with residue field $k$ and with maximal ideal $\mathfrak{m}$. Let $f_{1}$ and $f_{2}$ two $R$-valued points of $p$-Isog such that $f_{1} \otimes k$ and $f_{2} \otimes k$ are $\mu$-ordinary $p$-isogenies. Then the bi-infinitesimal parts of $f_{1} \otimes k$ and $f_{2} \otimes k$ (in the sense of (7.4)) are isomorphic. Hence it follows from $(7.5)(7)$ that the bi-infinitesimal parts of $f_{1} \otimes R / \mathrm{m}^{n+1}$ and $f_{2} \otimes R / \mathrm{m}^{n+1}$ are isomorphic for all $n \geq 0$.

(7.7) It follows that every point of $p$-Isog ${ }^{\mu}$ can be lifted to characteristic zero. More precisely there exists a canonical lift:

Let $k$ be a perfect field and let $f:(A, \iota, \lambda, \bar{\eta}) \longrightarrow\left(A^{\prime}, \iota^{\prime}, \lambda^{\prime}, \bar{\eta}^{\prime}\right)$ be a $\mu$-ordinary p-isogeny over $k$. It induces a homomorphism of $p$-divisible groups

$$
f\left[p^{\infty}\right]: X=A\left[p^{\infty}\right] \longrightarrow X^{\prime}=A\left[p^{\infty}\right]
$$

This homomorphism admits a decomposition $f\left[p^{\infty}\right]=f_{\text {et }} \times f_{\text {bi }} \times f_{\text {mult }}$ given by the decomposition of $X$ and $X^{\prime}$ into the product of its étale, bi-infinitesimal and multiplicative part. Each of the factors admits a unique lift to an isogeny over $W(k)$ : this is obvious for $f_{\text {et }}$ and $f_{\text {mult }}$ and it holds for $f_{\text {bi }}$ because of $(7.5)(7)$. The product of these lifts gives via the Serre-Tate theorem also a lifting of $f$ to a $p$-isogeny $\tilde{f}$ over $W(k)$. This lift will be called the canonical lift of $f$.

Its formation commutes with base change in the following sense: If $k_{1}$ is a perfect extension of $k$, denote by $f_{1}$ the extension of $f$ to $k_{1}$ and by $\tilde{f}_{1}$ the canonical lift of $f_{1}$ to $W\left(k_{1}\right)$. Then there is a canonical identification of $\tilde{f} \otimes_{W(k)} W\left(k_{1}\right)$ with $\tilde{f}_{1}$ where $W(k) \longrightarrow W\left(k_{1}\right)$ is induced from the inclusion $k \hookrightarrow k_{1}$.

(7.8) Theorem: Assume that $n$ is even. Then the $\mu$-ordinary locus of $p$-Isog $\otimes$ $\kappa\left(O_{E_{v}}\right)$ is dense.

Proof: Denote by $\mathcal{M}^{\text {nss }}$ the non-supersingular locus of the special fibre $\mathcal{M}$ of 
$\mathcal{M}_{\mathcal{I}, K^{p}}$. By (7.3) we know that the source and target morphism

$$
s, t: Y^{\mathrm{nss}} \longrightarrow \mathcal{M}^{\mathrm{nss}}
$$

are finite. By [We1] $\mathcal{M}^{\mu}$ is dense in $\mathcal{M}$. Hence every component of $Y^{\text {nss }} \backslash Y^{\mu}$ has dimension strictly less than the dimension of $\mathcal{M}$ which is equal to $n-1$. But every component of $Y^{\text {nss }}$ has dimension $\geq n-1$ (6.15), therefore $Y^{\mu}$ has to be dense in $Y^{\mathrm{nss}}$. It remains to show that no irreducible component of $Y$ is contained in $Y \backslash Y^{\mathrm{nss}}$. If that were the case we would find a point $y \in Y$ which has a neighborhood $U$ which is open in $Y$ and which is contained in the supersingular locus $Y \backslash Y^{\text {nss }}$. Let $\mathcal{M}^{\text {ss }}$ be the supersingular locus in $\mathcal{M}$. The closed immersion (7.5)

$$
(s, t): \operatorname{Spf}\left(\hat{\mathcal{O}}_{Y, y}\right)=\operatorname{Spf}\left(\hat{\mathcal{O}}_{U, y}\right) \longrightarrow \operatorname{Spf}\left(\hat{\mathcal{O}}_{\mathcal{M}, s(y)}\right) \times \operatorname{Spf}\left(\hat{\mathcal{O}}_{\mathcal{M}, t(y)}\right)
$$

would factorize through $\mathcal{S}=\operatorname{Spf}\left(\hat{\mathcal{O}}_{\mathcal{M}^{\mathrm{ss}}, s(y)}\right) \times \operatorname{Spf}\left(\hat{\mathcal{O}}_{\mathcal{M}^{\mathrm{ss}}, t(y)}\right)$. But by $(5.5)$ we have $\operatorname{dim}(\mathcal{S})=2[(n-1) / 2]$ which is strictly smaller than $n-1$ as $n$ is even. This is a contradiction to $(6.15)$.

(7.9) Corollary: Assume that $n$ is even.

(1) Every point of $p$-Isog $\otimes \kappa\left(E_{v}\right)$ can be lifted to characteristic zero.

(2) The scheme $p$-Isog $\otimes \kappa\left(E_{v}\right)$ is equi-dimensional of dimension $n-1$.

Proof: This follows from (7.8), (7.7) and (7.3).

\section{Hecke stratifications of the space of p-isogenies}

(8.1) Let $L$ be an unramified extension of $\mathbb{Q}_{p}$ and let $K_{L}$ be the unique hyperspecial subgroup of $G(L)$ such that $K_{L} \cap G\left(\mathbb{Q}_{p}\right)=K_{p}$ where $K_{p}$ is the stabilzer of $\Lambda$ in $V_{\mathbb{Q}_{p}}$. Let $M$ and $M^{\prime}$ be two $O_{B}$-invariant lattices of $V \otimes_{\mathbb{Q}_{p}} L$ which are selfdual up to a scalar. By a lemma of Kottwitz [Ko] there exists a $g \in G(L)$ such that $g M=M^{\prime}$. The residue class of $g$ in $K_{L} \backslash G(L) / K_{L}$ is uniquely determined and we denote it by $\operatorname{inv}_{L}\left(M, M^{\prime}\right)$. For $h \in G(L)$ we have $\operatorname{inv}_{L}\left(h M, h M^{\prime}\right)=\operatorname{inv}_{L}\left(M, M^{\prime}\right)$.

Sending an $L$-rational one-parameter subgroup $\lambda$ to the double coset of $\lambda(p)$ in $K_{L} \backslash G(L) / K_{L}$ defines a bijection between $G(L)$-conjugacy classes of one-parameter subgroups $\mathbb{G}_{m, L} \longrightarrow G_{L}$ and $K_{L} \backslash G(L) / K_{L}$. Hence we get an identification

$$
K_{L} \backslash G(L) / K_{L}=X_{*}(S) / \Omega(L)=\left(X_{*} / \Omega\right)^{\operatorname{Gal}\left(L^{\prime} / L\right)}
$$


where $S$ is an $L$-rational maximal split torus of $G_{L}$ and where $L^{\prime}$ is any extension of $L$ such that $G_{L^{\prime}}$ is split. Via this identification we consider $\operatorname{inv}_{L}\left(M, M^{\prime}\right)$ as the unique dominant coweight in $X_{*}$ which represents the corresponding element in $X_{*} / \Omega$.

We endow $X_{*} / \Omega$ with a partial order by saying $\lambda \Omega \leq \lambda^{\prime} \Omega$ if $\lambda \Omega$ is in the convex hull of $\lambda^{\prime} \Omega$ in $X_{*} \otimes \mathbb{R}$.

(8.2) Let $\bar{s} \longrightarrow p$-Isog be a geometric point in characteristic zero corresponding to a $p$-isogeny $f:(A, \iota, \lambda, \bar{\eta}) \longrightarrow\left(A^{\prime}, \iota^{\prime}, \lambda^{\prime}, \bar{\eta}^{\prime}\right)$. We get an induced map $T_{p}(f): T_{p}(A) \stackrel{\sim}{\longrightarrow}$ $T_{p}\left(A^{\prime}\right)$. Due to the determinant condition we can choose an $O_{B}$-linear symplectic similitude $T_{p}(A) \stackrel{\sim}{\longrightarrow} \Lambda$ (e.g. [RZ] 6.10) and via this isomorphism we consider $T_{p}(A)$ and $T_{p}\left(A^{\prime}\right)$ as lattices in $V_{\mathbb{Q}_{p}}$. Both are $O_{B}$-invariant and selfdual up to a scalar. Let $s \in S$ be the topological image of $\bar{s}$. Then

$$
\eta^{0}(s):=\operatorname{inv}_{\mathbb{Q}_{p}}\left(T_{p}(A), T_{p}\left(A^{\prime}\right)\right)
$$

is independent of the choice of $\bar{s}$ over $s$ and the choice of the isomorphism $T_{p}(A) \cong \Lambda$. Altogether we obtain a map

$$
\eta^{0}: p-\operatorname{Isog} \otimes E \longrightarrow\left(X_{*} / \Omega\right)^{\sigma}
$$

where $\sigma$ denotes the Frobenius automorphism. This map is clearly locally constant. Hence $p$-Isog $\otimes E$ is the union of open and closed subschemes $p$-Isog ${ }_{E}^{\alpha}$ with $\alpha \in\left(X_{*} / \Omega\right)^{\sigma}$.

(8.3) Let $p$-Isog ${ }^{\mu}$ be the $\mu$-ordinary locus of $p$-Isog $\otimes \kappa\left(E_{v}\right)$ and $s$ be a point of $p$-Isog ${ }^{\mu}$. Let $\bar{s}: \operatorname{Spec}(k) \longrightarrow p$-Isog ${ }^{\mu}$ be a geometric point over $s$ corresponding to a p-isogeny $f:(A, \iota, \lambda, \bar{\eta}) \longrightarrow\left(A^{\prime}, \iota^{\prime}, \lambda^{\prime}, \bar{\eta}^{\prime}\right)$ over $k=\kappa(\bar{s})$.

By (7.7) there exists a canonical lift $\tilde{f}$ of $f$ to $W(k)$. Denote by

$$
\alpha=T_{p}\left(\tilde{f}^{0}\right):\left(T_{p}\left(A^{0}\right), \iota,\langle,\rangle\right) \longrightarrow\left(T_{p}\left(A^{\prime 0}\right), \iota^{\prime},\langle,\rangle^{\prime}\right)
$$

the map on Tate modules which is induced by the generic fibre of $\tilde{f}$. Because of the determinant condition we can choose a $B$-linear symplectic similitude of $\left.\left(T_{p}\left(A^{0}\right), \iota,\langle\rangle,\right) \otimes \mathbb{Q}_{p}=\left(T_{p}\left(A^{\prime 0}\right), \iota^{\prime},\langle,\rangle^{\prime}\right) \otimes \mathbb{Q}_{p}\right)$ with the skew-hermitian $B$-module $\left(V_{\mathbb{Q}_{p}},\langle,\rangle_{\mathbb{Q}_{p}}\right)$. Hence we can consider $T_{p}\left(A^{0}\right)$ and $T_{p}\left(A^{\prime 0}\right)$ as $O_{B}$-lattices in $V_{\mathbb{Q}_{p}}$ which are selfdual up to a scalar.

By construction the induced homomorphism of $\tilde{f}$ on $p$-divisible groups splits into a product of a lift of the étale, the bi-infinitesimal, and the multiplicative part of $f$. 
Hence there exists an $h \in L\left(\mathbb{Q}_{p}\right)$ such that $h M=M^{\prime}$ where $L \subset G_{\mathbb{Q}_{p}}$ is the Levi subgroup defined in (1.5). Denote by ${ }_{L} K$ the intersection $K \cap L\left(\mathbb{Q}_{p}\right)$. The double coset

$$
\eta^{\mu}(s) \in{ }_{L} K \backslash L\left(\mathbb{Q}_{p}\right) /{ }_{L} K
$$

of $h$ is well defined and depends only on $s$. As in (8.1) we can consider $\eta^{\mu}(s)$ as an element of ${ }_{L} X_{*} /{ }_{L} \Omega$ where $\left({ }_{L} X^{*},{ }_{L} R^{*},{ }_{L} X_{*},{ }_{L} R_{*},{ }_{L} \Delta\right)$ is the based root datum of $L$ with Weyl group ${ }_{L} \Omega$. In fact $\eta^{\mu}(s)$ lies automatically in the $\sigma$-invariants of ${ }_{L} X_{*} / L \Omega$. Note further that $\eta^{\mu}(s)$ does not depend on the choice of $\bar{s}(7.7)$.

\section{(8.4) Proposition: The map}

$$
\eta^{\mu}: p-\operatorname{Isog}^{\mu} \longrightarrow{ }_{L} X_{*} /{ }_{L} \Omega
$$

is locally constant.

Proof : It suffices to show the following: Let $g:(A, \iota, \lambda, \bar{\eta}) \longrightarrow\left(A^{\prime}, \iota^{\prime}, \lambda^{\prime}, \bar{\eta}^{\prime}\right)$ be an $S$-valued point of $p$-Isog ${ }^{\mu}$ where $S$ is a $\kappa\left(O_{E_{v}}\right)$-scheme which is of the form $S=$ $\operatorname{Spec}(k[t]])$ for some algebraically closed field $k$. Let $s$ be the special point of $S$ and $t$ be a geometric pont of $S$ lying over the generic point of $S$. Then $\eta^{\mu}(s)=\eta^{\mu}(t)$. Denote by $f: \underline{X} \longrightarrow \underline{X}^{\prime}$ the associated morphism of $p$-divisible $\mathcal{I}$-modules. Denote by $G^{\prime}$ the reductive $\mathbb{Q}_{p}$-group $G^{\prime}=G L_{O_{K}}\left(V_{\mathbb{Q}_{p}}\right)$ and by $L^{\prime}$ the centralizer of $N(\mu)(1.5)$ in $G^{\prime}$. The inclusion $L \longrightarrow L^{\prime}$ induces a map ${ }_{L} X_{*} /{ }_{L} \Omega \longrightarrow L_{L^{\prime}} X_{*} / L_{L^{\prime}} \Omega$. It is easy to see that this map is injective. Hence it suffices to prove that the type of $f$ as a $p$-isogeny of $p$-divisible $O_{K}$-modules is locally constant. As the étale and the multiplicative rank of $X$ and $X^{\prime}$ are locally constant functions on $S$ we get a filtration of $p$-divisible $O_{K}$-modules

$$
0 \subset(X, \iota)_{\text {mult }} \subset(X, \iota)_{\inf } \subset(X, \iota)
$$

whose successive quotients are $(X, \iota)_{\mathrm{bi}}$ resp. $(X, \iota)_{\text {ét }}$, ditto for $\left(X^{\prime}, \iota^{\prime}\right)([\mathrm{Gr}]$ III, 7.4). We obtain an induced $p$-isogeny on the associated graded $p$-divisible $O_{K}$-modules

$$
\operatorname{gr}(f)=f_{\text {mult }} \times f_{\mathrm{bi}} \times f_{\text {ét }}
$$

and by definition the type of $f$ and that of $\operatorname{gr}(f)$ define the same function on $S$. Hence we can assume that $X$ and $X^{\prime}$ are products of their multiplicative, their biinfinitesimal and their étale part.

We set $k_{1}=\kappa(t)$. Let $\tilde{f}_{s}$ (resp. $\tilde{f}_{t}$ ) be the canonical lift of $f_{t}\left(\right.$ resp. $f_{s}$ ) to $W(\kappa(s))=W(k)$ (resp. to $\left.W\left(k_{1}\right)\right)$. It suffices to find a local $\operatorname{ring} R$ with $\left.R / p R=k[t]\right]$ together with $W(k)$-homomorphisms $R \longrightarrow W(k)$ and $R \longrightarrow W\left(k_{1}\right)$ and a $O_{K}$-linear 
p-isogeny $\tilde{f}$ over $R$ which lifts $f$ such that $\tilde{f} \otimes W(k)=\tilde{f}_{s}$ and $\tilde{f} \otimes W\left(k_{1}\right)=\tilde{f}_{t}$. For $R$ we take $W(k)[[T]]$. As homomorphism $R \longrightarrow W(k)$ (resp. $R \longrightarrow W\left(k_{1}\right)$ ) we take the unique $W(k)$-homomorphism which sends $T$ to zero (resp. to the Teichmüller representative of $t$ in $\left.W\left(k_{1}\right)\right)$. Our $\tilde{f}$ will be a product $\tilde{f}=\tilde{f}_{\text {ét }} \times \tilde{f}_{\text {bi }} \times \tilde{f}_{\text {mult }}$ where $\tilde{f}_{\text {? }}$ lifts $f_{\text {? }}$. To lift $f_{\text {ét }}$ and $f_{\text {mult }}$ is trivial, hence we have only to construct $\tilde{f}_{\text {bi }}$. Because of the uniqueness of lifts of $f_{s, \text { bi }}$ and $f_{t, \mathrm{bi}}(7.5)$, it suffices to construct a compatible system of lifts $\tilde{f}_{\mathrm{bi}, n}$ of $f_{\mathrm{bi}}$ to $W_{n}(k)[[t]]$. This can be done as in the proof of $(7.5)(7)$ using the theory of Dieudonné windows developed in [Zi3] (one can take as a frame ( $A=W(k)\left[[t], p^{n} A, \sigma\right)$ where $\sigma$ is the unique continuous ring endomorphism of $A$ with $\sigma(T)=T^{p}$ which induces on $W(k)$ the Frobenius).

(8.5) Let $k$ be a perfect field of characteristic $p$ and let $f:(X, \iota, \lambda) \longrightarrow\left(X^{\prime}, \iota^{\prime}, \lambda^{\prime}\right)$ be a homomorphism of $p$-divisible $\mathcal{I}$-modules which is induced by a $\mu$-ordinary $p$-isogeny over $k$. Denote by $\tilde{f}^{0}$ the generic fibre of the canonical lift of $f$ to $W(k)$. It is the product $\tilde{f}_{\text {ét }}^{0} \times \tilde{f}_{\mathrm{bi}}^{0} \times \tilde{f}_{\text {mult }}^{0}$ where $\tilde{f}_{\text {? }}^{0}$ is the generic fibre of the lift of $f_{\text {? }}$. Then the invariant factors of $T_{p}\left(\tilde{f}_{\text {ét }}^{0}\right)$ (resp. of $T_{p}\left(\tilde{f}_{\text {mult }}^{0}\right)$ ) in the sense of [BouA] chap. VII, $\S 4$, $\mathrm{n}^{o} 6$ are the same as the invariant factors of $T_{p}(f)$ (resp. of $T_{p}\left({ }^{t} f\right)$ ). This follows from (duality and):

Lemma: Let $S$ be a connected noetherian scheme and let $f: X \longrightarrow X^{\prime}$ be an isogeny of $p$-divisible groups. Assume that $X_{s}[p]$ is an étale group scheme for all $s \in S$. Then the invariant factors of $T_{p}\left(f_{\bar{s}}\right)$ are the same for all geometric points $\bar{s} \longrightarrow S$.

Proof: The functor $X \mapsto T_{p}\left(X_{\bar{s}}\right)$ sets up an equivalence between the category of étale $p$-divisible groups over $S$ and the category of finitely generated free $\mathbb{Z}_{p}$-modules with continuous $\pi_{1}(S, \bar{s})$-action and the latter category is independent of the choice of $\bar{s}$ up to inner automorphism.

(8.6) Altogether we obtain two decompositions

$$
\begin{aligned}
p \text {-Isog } \otimes E & =\coprod_{\alpha \in\left(X_{*} / \Omega\right)^{\sigma}} p-\operatorname{Isog}_{E}^{\alpha}, \\
p-\operatorname{Isog}^{\mu} & \left.=\coprod_{\gamma \in\left(L X_{*} / L\right.} p\right)^{\sigma} p-\operatorname{Isog}^{\mu, \gamma}
\end{aligned}
$$

into open and closed subschemes. 
(8.7) Proposition: Source and target morphism $p$-Isog ${ }^{\mu} \longrightarrow \mathcal{M}^{\mu}$ are finite locally free.

Proof: It suffices to prove this for the source morphism $s$ (by duality). We know already that the source morphism is finite (7.3). For every point $x$ of $\mathcal{M}$ the fibre $s^{-1}(\bar{x})$ of a geometric point $\bar{x}$ lying over $x$ is the spectrum of a finite $\kappa(\bar{x})$-algebra $A_{\bar{x}}$. As $\mathcal{M}^{\mu}$ is regular and in particular reduced, it suffices to show that the function $x \mapsto \operatorname{dim}_{\kappa(\bar{x})}\left(A_{\bar{x}}\right)$ is locally constant. From (7.2) we already know that $x \mapsto \# s^{-1}(\bar{x})$ is locally constant. Because of (8.4) it suffices to show: Let $\bar{y} \longrightarrow p$-Isog ${ }^{\mu, \gamma}$ be a geometric point and $\bar{x}=s(\bar{y})$ its image in $\mathcal{M}^{\mu}$. Then the $\kappa(\bar{x})$-dimension of $\mathcal{O}_{s^{-1}(\bar{x}), \bar{y}}=\hat{\mathcal{O}}_{s^{-1}(\bar{x}), \bar{y}}$ depends only on $\gamma$. But it follows from (7.5) and (7.6) that the isomorphism class of the $\kappa(\bar{x})$-algebra $\hat{\mathcal{O}}_{s^{-1}(\bar{x}), \bar{y}}$ depends only on $T_{p}(f)$ and $T_{p}\left({ }^{t} f\right)$ and in particular only on $\gamma(8.5)$.

(8.8) For any locally noetherian scheme $Y$ we denote by $\mathbb{Q}[Y]$ the $\mathbb{Q}$-vector space which has as a basis the components of $Y$. If $V \subset Y$ is an open dense subset we have a canonical identification $\mathbb{Q}[V]=\mathbb{Q}[Y]$ of $\mathbb{Q}$-vector spaces.

We will consider the cases $Y=p$-Isog $\otimes E$ and $Y=p$-Isog $\otimes \kappa\left(E_{\nu}\right)$. Reduction of cycles defines a map

$$
\operatorname{red}_{p}: \mathbb{Q}[p-\operatorname{Isog} \otimes E] \longrightarrow \mathbb{Q}\left[p-\operatorname{Isog} \otimes \kappa\left(E_{v}\right)\right]
$$

which associates to each component of $p$-Isog $\otimes E$ the intersection of its closure in $p$-Isog with $p$-Isog $\otimes \kappa\left(E_{v}\right)$

(8.9) Set $p$-Isog ${ }^{0}=p$-Isog $\otimes E$ and $\mathcal{M}^{0}=\mathcal{M} \otimes E$. Consider the morphism

$$
\text { comp: } p \text {-Isog }{ }^{0} \times_{t, \mathcal{M}^{0}, s} p \text {-Isog }{ }^{0} \longrightarrow p \text {-Isog }{ }^{0}, \quad(f, g) \mapsto g \circ f
$$

which is given by composition of $p$-isogenies. This morphism is finite étale because source and target morphism $s, t: p$-Isog ${ }^{0} \longrightarrow \mathcal{M}^{0}$ are finite étale, t. If $Z_{1}$ and $Z_{2}$ are two irreducible components of $p$-Isog ${ }^{0}$, we form the fibre product of

$$
\begin{aligned}
& \mathcal{M}^{0} \\
& \downarrow \Delta_{\mathcal{M}^{0}} \\
& Z_{1} \times Z_{2} \stackrel{t, s}{\longrightarrow} \mathcal{M}^{0} \times \mathcal{M}^{0} .
\end{aligned}
$$

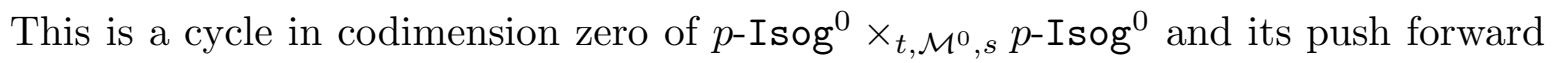
via comp is an element in $\mathbb{Q}\left[p\right.$-Isog $\left.{ }^{0}\right]$. This defines the structure of a $\mathbb{Q}$-algebra on $\mathbb{Q}\left[p-\operatorname{Isog}^{0}\right]$. 
In the same way (by replacing $?^{0}$ by $?^{\mu}$ and using (8.7)) we get a multiplication on $\mathbb{Q}\left[p\right.$-Isog $\left.{ }^{\mu}\right]$. If $n$ is even, (7.8) implies that we have $\mathbb{Q}\left[p-\operatorname{Isog} \otimes \kappa\left(E_{\nu}\right)\right]=\mathbb{Q}\left[p-\operatorname{Isog}^{\mu}\right]$. Hence $\mathbb{Q}\left[p\right.$-Isog $\left.\otimes \kappa\left(E_{\nu}\right)\right]$ obtains the structure of a $\mathbb{Q}$-algebra such that $\operatorname{red}_{p}(8.8)$ becomes a homomorphism of $\mathbb{Q}$-algebras.

(8.10) Assume that $n$ is even. In [We2] $\S 4$ a $\mathbb{Q}$-algebra homomorphism

$$
h^{0}: \mathcal{H}_{\mathbb{Q}}\left(G\left(\mathbb{Q}_{p}\right) / / K_{p}\right)_{0} \longrightarrow \mathbb{Q}[p-\operatorname{Isog} \otimes E]
$$

is constructed which sends the characteristic function of a $K_{p}$-double coset $\alpha$ of $G\left(\mathbb{Q}_{p}\right)$ to the corresponding stratum $(p \text {-Isog } \otimes E)^{\alpha}$. This stratum consists of those points $f$ in $p$-Isog $\otimes E$ where $f$ is given on Tate modules by $\alpha$ using the identification (1.6.1) (see loc. cit. for the precise definition).

In $\mathbb{Q}\left[p\right.$-I $\left.\operatorname{sog} \otimes \kappa\left(E_{v}\right)\right]$ we have the element Frob corresponding to relative Frobenius isogenies as in (1.7.1).

The principal result is now:

Main Theorem: We consider $\mathbb{Q}\left[p\right.$-Isog $\left.\otimes \kappa\left(E_{v}\right)\right]$ via $\operatorname{red}_{p} \circ h^{0}$ as an algebra over $\mathcal{H}_{\mathbb{Q}}(G)$. Then we have

$$
H_{G,\{\mu\}}(\text { Frob })=0 .
$$

(8.11) To prove the main theorem we use the stratification of $p$-Isog ${ }^{\mu}$ indexed by elements in ${ }_{L} X_{*} /{ }_{L} \Omega$ and (8.3). We define a $\mathbb{Q}$-algebra homomorphism

$$
\bar{h}: \mathcal{H}_{\mathbb{Q}}(L)_{0} \longrightarrow \mathbb{Q}\left[p-\operatorname{Isog}^{\mu}\right]=\mathbb{Q}\left[p-\operatorname{Isog} \otimes \kappa\left(E_{v}\right)\right]
$$

as in [We2] $\S 5$ where $\mathcal{H}_{\mathbb{Q}}(L)_{0}$ denotes again the subalgebra of functions with support in $\operatorname{End}(\Lambda)$. It is defined as the map which sends the characteristic function of an ${ }_{L} K$-double coset (corresponding to a $\gamma \in{ }_{L} X_{*} /{ }_{L} \Omega$ ) to the cycle of the corresponding stratum divided by a constant $a(\gamma)$. This constant is defined as the formal multiplicity of the geometric fibres of the source morphism $p$-Isog ${ }^{\mu} \longrightarrow \mathcal{M}^{\mu}$. It depends only on $\gamma$ by (8.7).

Further denote by

$$
\dot{\mathcal{S}}: \mathcal{H}_{\mathbb{Q}}(G) \longrightarrow \mathcal{H}_{\mathbb{Q}}(L)
$$

the twisted Satake homomorphism defined in [We2] $§ 1$. As in [FC] VII,4 we have a 
commutative diagram

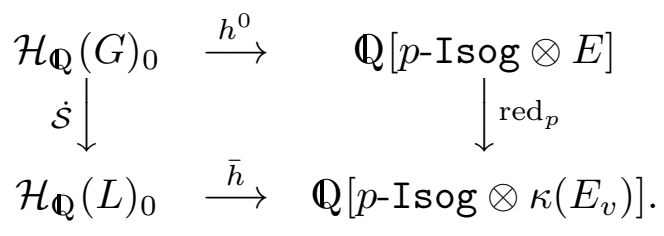

(8.12) Proof of the main theorem (8.10): Recall that we have defined in (1.5) an element $\{N(\mu)\} \in\left(X_{*} / L \Omega\right)^{\sigma}$. This is the same as the element $[\mu]$ defined in [We2] 2.4. Let $g \in \mathcal{H}_{\mathbb{Q}}(L)$ be the corresponding characteristic function (8.11). Let $H_{G,\{\mu\}} \in \mathcal{H}_{\mathbb{Q}}(G)[X]$ be the Hecke polynomial defined in loc. cit. 2.6. We regard $\mathcal{H}_{\mathbb{Q}}(L)$ via the twisted Sataka homomorphism $\dot{\mathcal{S}}$ as an algebra over $\mathcal{H}_{\mathbb{Q}}(G)$. By loc. cit. 2.9 we have

$$
H_{G,\{\mu\}}(g)=0 .
$$

Hence by (8.11.1) it suffices to show that the element $g$ is sent to the element Frob in $\mathbb{Q}\left[Y \otimes \kappa\left(E_{v}\right)\right]$ via $\bar{h}$ where Frob is the relative Frobenius isogeny with respect to $\kappa\left(E_{v}\right)$.

For this we use the notations of (1.3). We may assume (to simplify notations) that $n>2$, hence $n \geq 4$ as $n$ is even, as the case $n=2$ is the classical case (of elliptic curves). Then $E$ is a quadratic imaginary extension of $\mathbb{Q}$ and we have $E_{v}=K$. We have $B \otimes \mathbb{Q}_{p}=M_{m}(K)$ und using Morita equivalence we can assume that $m=1$. Hence we can identify $G_{\mathbb{Q}_{p}}$ with $G U\left(V_{\mathbb{Q}_{p}}, \beta\right)$ where $\beta: V_{\mathbb{Q}_{p}} \times V_{\mathbb{Q}_{p}} \longrightarrow K$ is the skewhermitian form such that its composition with $\operatorname{Tr}_{K / \mathbb{Q}_{p}}$ is $\langle,\rangle_{\mathbb{Q}_{p}}$. Using the canonical embedding $G_{\mathbb{Q}_{p}} \hookrightarrow G S p\left(V_{\mathbb{Q}_{p}},\langle,\rangle_{\mathbb{Q}_{p}}\right)$ we identify $X_{*}$ with

$$
\left\{\left(x_{i}\right) \in \mathbb{Z}^{2 n} \mid x_{i}+x_{2 n+1-i}=\text { const for all } i\right\}
$$

and $\Omega$ with $S_{n}$ which acts on $X_{*}$ by permuting simlutaneously $\left(x_{1}, \ldots, x_{n}\right)$ and $\left(x_{2 n}, \ldots, x_{n+1}\right)$. The element $\{\mu\} \in X_{*} / \Omega$ is given by the class of $\left(1,0^{n-1}, 1^{n-1}, 0\right)$ because of our assumption on the signature. Further the nontrivial element $\sigma$ in $\operatorname{Gal}\left(K / \mathbb{Q}_{p}\right)$ acts by sending $\left(x_{i}\right)_{1 \leq i \leq 2 n}$ to $\left(x_{n+1}, \ldots, x_{2 n}, x_{1}, \ldots, x_{n}\right)$. Hence the norm of $\{\mu\}$ is given by

$$
N(\{\mu\})=\left(2,1^{n-2}, 0,2,1^{n-2}, 0\right) \in X_{*} / L \Omega .
$$

But this is precisely the type of $V^{2}$ on a $\mu$-ordinary unitary Dieudonné module (4.10). As Frob corresponds to $V^{2}$ via covariant Dieudonné theory, we see that Frob is the cycle in $\mathbb{Q}\left[p\right.$-Isog $\left.\otimes \kappa\left(E_{v}\right)\right]$ corresponding to $\gamma=N(\{\mu\})$. Further the definition of $a(\gamma)$ (8.11) shows that $a(N(\{\mu\})$ is equal to one which proves the claim. 


\section{Bibliography}

[BR] D. Blasius, J.D. Rogawski: Zeta functions of Shimura varieties, in: U. Jannsen, S. Kleiman, J.-P. Serre (ed.): Motives 2, Proc. Symp. Pure Math. 55 (1994), 447-524.

[BouA] N. Bourbaki: Algebra, chap. IV-VII, Springer (1988).

[De] P. Deligne: Variétés de Shimura, in A. Borel, W. Casselman (ed.): Automorphic forms, representations, and L-functions 2, Proc. Symp. Pure Math. 33 (1977), 247-289.

[Gr] A. Grothendieck: "Groupes de Barsotti-Tate et cristaux de Dieudonné," Presses de l'Université de Montréal, 1974.

[Ill] L. Illusie: Complexe de de Rham-Witt et cohomologie cristalline, Ann. Sci. École Norm. Sup. (4) 12 (1979), 501-661.

[Ko] R. Kottwitz: Points on some Shimura varieties over finite fields, J. AMS 5 (1992), 373-444.

[Kr] H. Kraft: Kommutative algebraische p-Gruppen (mit Anwendungen auf pdivisible Gruppen und abelsche Varietäten), lecture manuscript, Universität Bonn, 1975.

[Laz] M. Lazard: Commutative formal groups, Lecture Notes in Mathematics 443, Springer-Verlag, Berlin-New York, 1975.

[LM] G. Laumon, L. Moret-Bailly: "Champs algébriques", Ergebnisse der Mathematik und ihrer Grenzgebiete (3. Folge) 39, Springer (2000).

[Mo] B. Moonen: Group schemes with additional structure and Weyl group cosets, in "Moduli of Abelian Varieties" (ed. by C. Faber, G. van der Geer, F. Oort), Progress in Mathematics 195, Birkhäuser (2001).

[Ra] M. Raynaud: Schémas en groupes de type $(p, \ldots, p)$, Bull. Soc. Math. France 102 (1974), 241-280.

[RZ] M. Rapoport, T. Zink: Period Spaces for p-divisible Groups, Annals of Mathematics Studies 141, Princeton University Press, Princeton (1996).

[We1] T. Wedhorn: Ordinariness in good reductions of Shimura varieties of PELtype, Ann. Sci. de l'ENS 32 (1999), 575 - 618.

[We2] T. Wedhorn: Congruence relations for some Shimura varieties, J. reine angewandte Math. (Crelle) 524 (2000), 43-71.

[We3] T. Wedhorn: The dimension of Oort strata of Shimura varieties of PEL-type, in "Moduli of Abelian Varieties" (ed. by C. Faber, G. van der Geer, F. Oort), 
Progress in Mathematics 195, Birkhäuser (2001).

[Zi1] T. Zink: Cartiertheorie kommutativer formaler Gruppen, Teubner-Texte zur Mathematik 68, Leipzig (1984).

[Zi2] T. Zink: Cartier Theory and Crystalline Dieudonné Theory, preprint Bielefeld (1998).

[Zi3] T. Zink: Windows for Displays of p-divisible Groups, in "Moduli of Abelian Varieties" (ed. by C. Faber, G. van der Geer, F. Oort), Progress in Mathematics 195, Birkhäuser (2001). 